\title{
Theoretical Analysis for a Class of Rheonomous Affine Constraints on Configuration Manifolds-Part II: Foliation Structures and Integrating Algorithms
}

\author{
Tatsuya Kai \\ Department of Applied Electronics, Faculty of Industrial Science and Technology, \\ Tokyo University of Science, Chiba 278-8510, Japan \\ Correspondence should be addressed to Tatsuya Kai, kai@te.noda.tus.ac.jp \\ Received 6 May 2012; Revised 3 July 2012; Accepted 4 July 2012 \\ Academic Editor: Wei-Chiang Hong
}

Copyright (C) 2012 Tatsuya Kai. This is an open access article distributed under the Creative Commons Attribution License, which permits unrestricted use, distribution, and reproduction in any medium, provided the original work is properly cited.

\begin{abstract}
This paper investigates foliation structures of configuration manifolds and develops integrating algorithms for a class of constraints that contain the time variable, called $A$-rheonomous affine constrains. We first present some preliminaries on the $A$-rheonomous affine constrains. Next, theoretical analysis on foliation structures of configuration manifolds is done for the respective three cases where the $A$-rheonomous affine constrains are completely integrable, partially integrable, and completely nonintegrable. We then propose two types of integrating algorithms in order to calculate independent first integrals for completely integrable and partially integrable $A$-rheonomous affine constrains. Finally, a physical example is illustrated in order to verify the availability of our new results.
\end{abstract}

\section{Introduction}

Recent years, interest on nonholonomic systems [1-5] has been higher in the various research fields such as analytical mechanics, control theory, robotics, among others. Nonholonomic systems are defined as systems which are subject to some nonintegrable constraints and behaviors of the systems have to satisfy the constraints. Various examples of nonholonomic systems can be easily found, for example, mobile cars [6, 7], trailers [8,9], space robots $[10,11]$, acrobat robots $[12,13]$, a rolling ball or coin on a plain [1], underactuated manipulators [14-16], among others. In a lot of researches on nonholonomic control systems, linear constraints, which are represented in the form: $B(q) \dot{q}=0, q \in \mathbf{R}^{n}, B(q) \in \mathbf{R}^{(n-m) \times n}$, have been mainly considered so far. The class of the linear constraints contains many examples 
such as mobile cars, a ball or a coin on a plain, hopping robots [14, 15], among others. It is well known that integrability and nonintegrability of the linear constraints can be determined by using well-known Frobenius' theorem [17, 18], and there is a strong relationship between them and accessibility of nonlinear control systems with the linear constraints [4, 5, 7, 17-21]. Moreover, for kinematic and dynamic systems subject to the linear constraints, the following results are also well known: (i) their linear approximated systems are uncontrollable, (ii) they are locally controllable, but not locally asymptotically stabilizable by any nonlinear smooth state feedback laws from Brockett's theorem [4, 22]. Hence, a lot of work have been done to overcome the difficulties until now.

However, the class of the linear constraints does not cover all of the nonholonomic systems. There exists a larger class of constraints and it is called scleronomous affine constraints, which are represented as $A(q)+B(q) \dot{q}=0, q \in \mathbf{R}^{n}, A(q) \in \mathbf{R}^{n-m}, B(q) \in \mathbf{R}^{(n-m) \times n}$. The terminology "scleronomous" means "time-invariant" and is mainly used for constraints. A space robots with an initial angular momentum (Figure 1(a)), a boat or a ship on a running river (Figure 1(b)), a ball or a coin on a rotating table (Figures 1(c) and 1(d)) are typical examples of systems subject to the scleronomous affine constraints. Compared to researches on the linear constraints, ones on the scleronomous affine constraints are quite few, but we have focused on the scleronomous affine constraints and derived various results from the viewpoints of both mathematics and nonlinear control theory [23-32]. In work on the scleronomous affine constraints, the following new results, which are beyond the results on the linear constraints mentioned above, have been obtained: (a) complete nonintegrability (nonholonomicity) of the scleronomous affine constraints is equivalent to strong accessibility of systems, (b) linear approximated systems of original systems with scleronomous affine constraints are controllable under some conditions, (c) some systems with scleronomous affine constraints satisfy Brockett's condition and hence there is a possibility of local asymptotic stabilizability by nonlinear smooth state feedback laws. Furthermore, we can consider a larger class of constraints which contains the time variable, that is to say, rheonomous constraints, and a boat or a ship on a running river with a time-varying stream (Figure 1(b)) and a coin or a ball on a rotating table at a timevarying angular velocity (Figures 1 (c) and 1(d)) are expected to be subject to rheonomous constraints. Since rheonomous constraints contain the time variable explicitly, it is more difficult to analyze and control systems subject to them in comparison with the scleronomous constraints case. To make matters worse, the results on scleronomous constraints cannot be utilized for rheonomous constraints, hence, we need fundamental theory for rheonomous constraints to consider analysis and control of systems with them. As a matter of course, such rheonomous constraints have been already treated in [33-37] by using various mathematical tools, however, fundamental properties for them such as integrability/nonintegrability and foliation structures of configuration manifolds have not been derived in these work.

This is the second paper of a series of papers that deal with a class of rheonomous constraints, called A-rheonomous affine constraints defined on configuration manifolds. The $A$-rheonomous affine constraints are represented in the form $A(t, q)+B(q) \dot{q}=0, q \in$ $\mathbf{R}^{n}, A(t, q) \in \mathbf{R}^{n-m}, B(q) \in \mathbf{R}^{(n-m) \times n}$, that is to say, affine constraints whose term $A$ contains the time variable $t$. The class of the $A$-rheonomous affine constraints is larger than the scleronomous affine constraints and contains some physical and mechanical examples as shown in Figure 2. In the first paper [38], we show some fundamental properties of the $A$-rheonomous affine constraints and derive some necessary and sufficient conditions on integrability/nonintegrability for them. On the other hand, in this second paper, we will focus on analysis of foliation structures of configuration manifolds with the $A$-rheonomous 


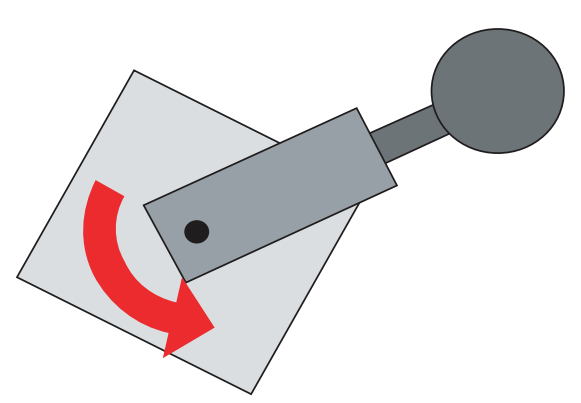

(a) Space robot with initial angular momentum

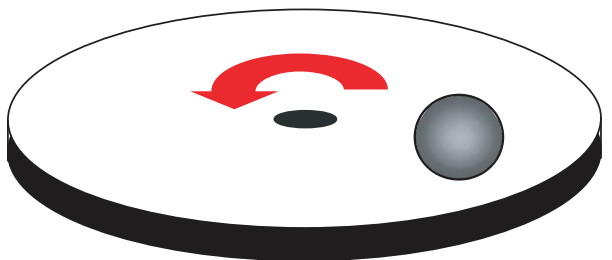

(c) Ball on rotating table

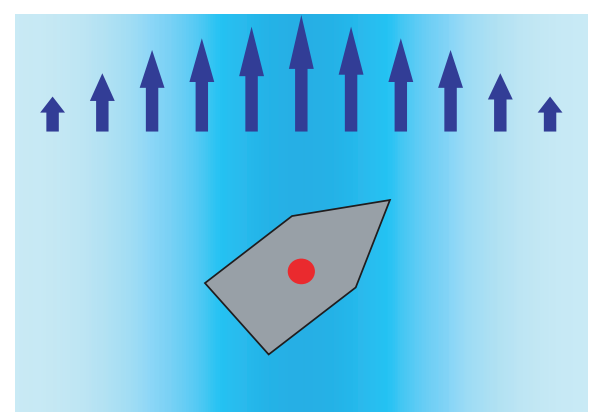

(b) Boat on running river

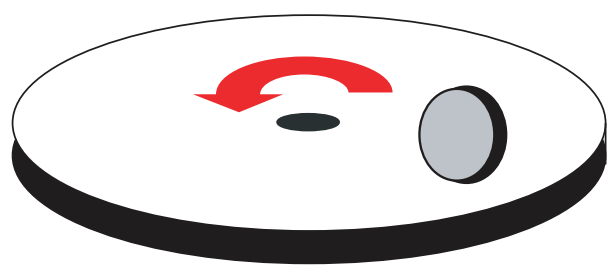

(d) Coin on rotating table

Figure 1: Examples of systems subject to affine constraints.

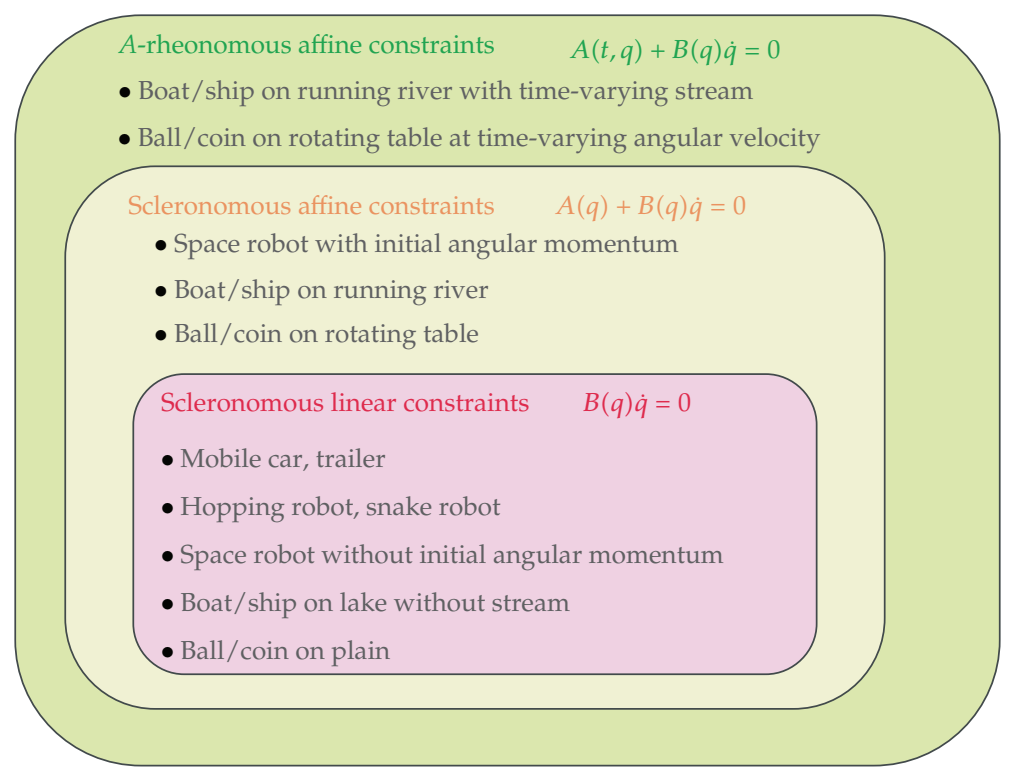

Figure 2: Classes of constraints and examples.

affine constraints, and development of integrating algorithms to calculate independent first integrals for integrable $A$-rheonomous affine constraints.

The outline of this paper is as follows. First, we give some preliminaries on the $A$ rheonomous affine constraints in Section 2. In Section 3, we next investigate the relationships between the $A$-rheonomous affine constraints and foliation structures of configuration manifolds in the respective three cases: complete integrability, partial integrability, and 
complete nonintegrability. Then, in Section 4, we develop two types of integrating algorithms to calculate independent first integrals for completely integrable and partially integrable $A$ rheonomous affine constraints. Moreover, theoretical analysis of the algorithms is also shown. Finally, we illustrate a physical example, a pendulum with a time-varying elastic string, in order to verify the availability of our new results in Section 5. Throughout this paper, manifolds, submanifolds, functions, vector fields, distributions, and differential forms are all assumed to be smooth.

\section{Preliminaries}

This section presents some preliminaries on rheonomous affine constraints which we deal with throughout this paper. See also the first paper [38] for more details. First, we define rheonomous affine constraints and explain geometric representation of them. We denote the time variable by $t \in \mathbf{R}$ and a time interval by $I \subset \mathbf{R}$. Let $Q$ be an $n$-dimensional configuration manifold and $q=\left[\begin{array}{lll}q_{1} & \cdots & q_{n}\end{array}\right]^{\top} \in \mathbf{R}^{n}$ be a local coordinate of $Q$. Associated with $q$, we refer $\dot{q}=\left[\begin{array}{lll}\dot{q}_{1} & \cdots & \dot{q}_{n}\end{array}\right]^{\top} \in T_{q} Q$ as a tangent vector field.

Throughout this paper, we deal with A-rheonomous affine constraints:

$$
A_{i}(t, q)+B_{i 1}(q) \dot{q}_{1}+\cdots+B_{i n}(q) \dot{q}_{n}=0, \quad i=1, \ldots, n-m
$$

Note that the coefficients $A_{i}(i=1, \ldots, n-m)$ in (2.1) depend on the time variable $t$. We rewrite (2.1) as

$$
A(t, q)+B(q) \dot{q}=0
$$

where a rheonomous affine term $A(t, q) \in \mathbf{R}^{n-m}$ is a vector-valued function whose $i$ th entry is $A_{i}(t, q)$, and $B(q)$ is a matrix-valued function whose $i j$ th entry is $B_{i j}(q)$. It must be noted that the class of the $A$-rheonomous affine constraints contains some important examples of mechanical systems as mentioned in Section 1. We here assume a sufficient condition on independency of the $A$-rheonomous affine constraints (2.2) as follows.

Assumption 2.1. The coefficient matrix $B(q)$ of the $A$-rheonomous affine constraints (2.2) has a row full-rank at any point $q \in Q$, that is,

$$
\operatorname{rank} B(q)=n-m, \quad \forall q \in Q
$$

holds.

Now, we consider a classification method of all the points in the configuration manifold $Q$ in terms of the rheonomous affine term $A(t, q)$ in the $A$-rheonomous affine constraints (2.2) by the following definition.

Definition 2.2 (see [38]). For the rheonomous affine term $A(t, q)$ of the $A$-rheonomous affine constraints (2.2) and a time interval $I \subset \mathbf{R}$, a point $q \in Q$ such that $A(t, q) \neq 0$, for all $t \in I$, holds is called a rheonomous affine regular point. On the other hand, a point $q \in Q$ such that $A(t, q)=0$, for all $t \in I$, holds is called $a$ rheonomous affine equilibrium point. 


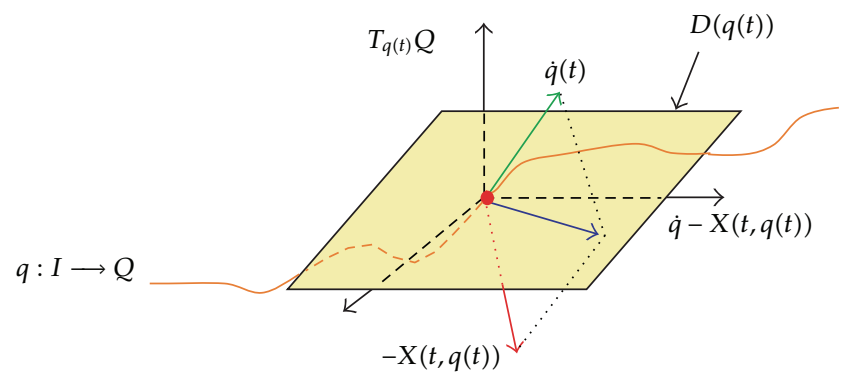

Figure 3: Geometric representation of $A$-rheonomous affine constraints.

Note that from Definition 2.2, each point in $Q$ is classified into either a rheonomous affine regular point or a rheonomous affine equilibrium point. Next, we explain geometric representation and some fundamental properties for the $A$-rheonomous affine constraints, which are originally obtained in the first paper [38]. From (2.3) in Assumption 2.1, the $n-m$ row vectors of $B(q)$ in the $A$-rheonomous affine constraints (2.2) are independent of each other at any point $q \in Q$. So, we consider $m$ vectors which are independent of each other and annihilators of the $n-m$ row vectors of $B(q)$, and denote them by $Y_{1}, \ldots, Y_{m}$ as vector fields on $Q$. Moreover, we also denote a space spanned by $Y_{1}, \ldots, Y_{m}$, that is, a distribution on $Q$ by

$$
D:=\operatorname{span}\left\{Y_{1}, \ldots, Y_{m}\right\}
$$

Since the basial vectors of $D: Y_{1}, \ldots, Y_{m}$ are independent of each other at any point $q \in Q, D$ is a nonsingular distribution, that is,

$$
\operatorname{dim} D(q)=m, \quad \forall q \in Q
$$

holds. A curve $q: I \rightarrow Q$ is said to be satisfied the A-rheonomous affine constraints (2.2) if for a vector field on $Q: X$ and the generalized velocity of $q: \dot{q} \in T_{q(t)} Q$ :

$$
\dot{q}(t)-X(t, q(t)) \in D(q(t)), \quad \forall t \in I,
$$

holds as shown in Figure 3. We call this a rheonomous affine vector field.

We then show an important characteristic of the rheonomous affine vector field $X$ as the next proposition.

Proposition 2.3 (see [38]). For the A-rheonomous affine constraints (2.2), the component of the rheonomous affine vector field $X$, and a time interval $I \subset \mathbf{R}$ :

$$
A(t, q)+B(q) X(t, q)=0, \quad \forall q \in Q, \forall t \in I,
$$

holds. 
Under the preliminaries shown above, we define the geometric representation method for the $A$-rheonomous affine constraints as the following definition.

Definition 2.4 (see [38]). The A-rheonomous affine constraints (2.2) are geometrically represented by a pair $(D, X)$, where $D$ is an $m$-dimensional distribution defined by (2.4) and $X$ is called a rheonomous affine vector field and satisfies (2.7).

In order to derive some theoretical analysis on $A$-rheonomous affine constraints (2.2), the geometric representation of them is quite important. For the geometric representation of the A-rheonomous affine constraints shown in Definition 2.4, the next proposition can be derived.

Proposition 2.5 (see [38]). For the geometric representation of the A-rheonomous affine constraints (2.2): $(D, X)$ and a time interval $I \subset \mathbf{R}$, a point $q \in Q$ is a rheonomous equilibrium point if and only if

$$
X(t, q(t)) \in D(q(t)), \quad \forall t \in I
$$

holds. On the other hand, a point $q \in Q$ is a rheonomous regular point if and only if

$$
X(t, q(t)) \notin D(q(t)), \quad \forall t \in I
$$

holds.

Finally, we explain the rheonomous bracket which is a new operator and originally introduced in the first paper [38]. Similar to the first paper [38], the rheonomous bracket also plays important roles in this second paper. The Lie bracket of two vector fields $Z, W$ is defined as an operator defined on $T Q$ :

$$
[Z, W]:=\frac{\partial W}{\partial q} Z-\frac{\partial Z}{\partial q} W
$$

Based on the Lie bracket above, the rheonomous bracket is defined as follows.

Definition 2.6 (see [38]). For the vector fields defined on $Q$ of the geometric representation of the $A$-rheonomous affine constraints (2.2): $X, Y_{1}, \ldots, Y_{m}$, the rheonomous bracket is an operator: $\langle\cdot, \cdot\rangle: T Q \times T Q \rightarrow T Q$ that satisfies the next three properties.

(a) For a rheonomous affine vector field $X$,

$$
\langle X, X\rangle=0
$$

holds. 
(b) $D_{0}$ is defined as a set of vector fields that consists of $Y_{1}, \ldots, Y_{m}$ and iterated rheonomous brackets of $X, Y_{1}, \ldots, Y_{m}$ and does not contain $X$. For a rheonomous affine vector field $X$ and a vector field $Z \in D_{0}$,

$$
\begin{aligned}
& \langle X, Z\rangle=\frac{\partial Z}{\partial t}+[X, Z], \quad Z \in D_{0} \\
& \langle Z, X\rangle=-\frac{\partial Z}{\partial t}+[Z, X], \quad Z \in D_{0}
\end{aligned}
$$

hold.

(c) For two vector fields $Z, W \in D_{0}$,

$$
\begin{gathered}
\langle Z, Z\rangle:=0, \quad Z \in D_{0}, \\
\langle Z, W\rangle:=[Z, W], \quad Z, W \in D_{0}
\end{gathered}
$$

hold.

Note that, in Definition 2.6, the rheonomous affine vector field $X$ is perceived as special, and this yields an additional term for time differential of a vector field as shown in the property (b). It is also noted that the rheonomous bracket is equivalent to the normal Lie bracket for scleronomous affine constraints, that is, constraints that do not contain the time variable explicitly. Moreover, the following proposition indicates that the rheonomous bracket has the important characteristics in common with the normal Lie bracket.

Proposition 2.7 (see [38]). For the vector fields on the geometric representation of the A-rheonomous affine constraints (2.2): $X, Y_{1}, \ldots, Y_{m}$ and the set of iterated vector fields of them: $D_{0}$, the following properties (a), (b), and (c) hold.

(a) Bilinearlity:

$$
\begin{gathered}
\langle X, a Z+b W\rangle=a\langle X, Z\rangle+b\langle X, W\rangle \\
\langle a Z+b W, X\rangle=a\langle Z, X\rangle+b\langle W, X\rangle, \quad Z, W \in D_{0} .
\end{gathered}
$$

(b) Skew-symmetry:

$$
\langle X, Z\rangle=-\langle Z, X\rangle, \quad Z, W \in D_{0} .
$$

(c) Jacobi's identity:

$$
\langle\langle X, Z\rangle, W\rangle+\langle\langle Z, W\rangle, X\rangle+\langle\langle W, X\rangle, Z\rangle=0, \quad Z, W \in D_{0}
$$




\section{Foliation Structures of Configuration Manifolds}

\subsection{Completely Integrable Case}

In this section, we investigate foliation structures of configuration manifolds for the respective three cases where the $A$-rheonomous affine constraints are completely integrable, partially integrable, and completely nonintegrable. It is quite essential to comprehend foliation structures of configuration manifolds in terms of model reduction theory, accessibility analysis of nonholonomic control systems, and so on.

First, we consider the complete integrability case. We now define a smallest and involutive time-varying distribution $C_{0}(t, q)$ which contains $Y_{1}, \ldots, Y_{m}$ and iterated rheonomous brackets of them, and satisfies $\langle X, W\rangle \in C_{0}$, for all $W \in C_{0}$, that is to say, $C_{0}$ is spanned by all the rheonomous brackets of $X, Y_{1}, \ldots, Y_{m}$ with the exception of $X$. In addition, we also define a smallest and involutive time-varying distribution $C(t, q)$ which contains all the iterated rheonomous brackets of $X, Y_{1}, \ldots, Y_{m}$. We here show necessary and sufficient conditions on complete integrability for the $A$-rheonomous affine constraints (2.2), which are originally derived in the first paper [38].

Theorem 3.1 (see [38]). For the A-rheonomous affine constraints (2.2) defined on an $n$-dimensional manifold $Q$ and a time interval I $\subset \mathbf{R}$, the following statements ( $a)-(c)$ are equivalent to each other. If they hold, the A-rheonomous affine constraints (2.2) are said to be completely integrable or completely holonomic.

(a) There exist $n-m$ independent first integrals of the A-rheonomous affine constraints (2.2): $h_{1}(t, q), \ldots, h_{n-m}(t, q)$ such that

$$
\begin{gathered}
\frac{\partial h_{i}(t, q)}{\partial t}+X(t, q) h_{i}(t, q)=0, \quad i=1, \ldots, n-m, \forall q \in Q, \forall t \in I, \\
Z(t, q) h_{i}(t, q)=0, \quad \forall Z \in D, i=1, \ldots, n-m, \forall q \in Q, \forall t \in I, \\
d h_{1} \wedge \cdots \wedge d h_{n-m}(t, q) \neq 0, \quad \forall q \in Q, \quad \forall t \in I,
\end{gathered}
$$

hold, where $d$ is the exterior differential operator defined on $Q$.

(b) There exists an m-dimensional time-varying integral manifold $S^{m}\left(t, q^{0}\right)$ of the $A$ rheonomous affine constraints (2.2) determined by an initial point $q^{0} \in Q$ such that

$$
T_{q} S^{m}\left(t, q^{0}\right)=D(t, q), \quad \forall q \in S^{m}\left(t, q^{0}\right), \forall t \in I,
$$

holds.

(c) For a smallest and involutive time-varying distribution $C_{0}$,

$$
\operatorname{dim} C_{0}(t, q)=m, \quad \forall q \in Q, \forall t \in I,
$$

holds.

From the statement (b) in Theorem 3.1, we can see that there exists an $m$-dimensional time-varying integral manifold in the $n$-dimensional configuration manifold $Q$. It is expected 
that this time-varying integral manifold creates a foliation structure in $Q$. Hence, we investigate the foliation structure with much circumstance. Now, we show the following lemma, which is useful for the proof of the main theorem.

Lemma 3.2. Assume that the A-rheonomous affine constraints (2.2) are completely integrable. Onedenotes a set of rheonomous affine regular points by

$$
U_{a}^{m}:=\{q \in Q \mid A(t, q) \neq 0, \forall t \in I\},
$$

and a set of rheonomous affine equilibrium points by

$$
U_{b}^{m}:=\{q \in Q \mid A(t, q)=0, \forall t \in I\},
$$

where $I \subset \mathbf{R}$ is a time interval. Then, the following statements ( $a$ ) and (b) hold.

(a) For $U_{a}^{m}$ and $U_{b}^{m}$,

$$
U_{a}^{m} \cap U_{b}^{m}=\phi, \quad U_{a}^{m} \cup U_{b}^{m}=Q
$$

hold, where $\phi$ denotes the empty set.

(b) For a neighborhood $V_{b}^{m} \subset U_{b}^{m}$ of a rheonomous affine equilibrium point $q^{e} \in U_{b}^{m}$,

$$
\operatorname{dim} V_{b}^{m} \geq m
$$

holds.

Proof. Since each point in $Q$ is classified into either a rheonomous affine regular point or a rheonomous affine equilibrium point from Definition 2.2, the statement (a) is determinately satisfied. Next, since a rheonomous affine equilibrium point $q^{e} \in U_{b}^{m}$ satisfies from Proposition 2.5, we can represent $X\left(t, q^{e}\right)$ as

$$
X\left(t, q^{e}\right)=\beta_{1}\left(t, q^{e}\right) Y_{1}\left(q^{e}\right)+\cdots+\beta_{m}\left(t, q^{e}\right) Y_{m}\left(q^{e}\right)
$$

with $m$ functions $\beta_{1}(t, q), \ldots, \beta_{m}(t, q)$. Consider $n-m$ vector fields $Y_{m+1}, \ldots, Y_{n}$ which are independent of each other and are orthogonal to $Y_{1}, \ldots, Y_{m}$. Calculating inner products of $X\left(t, q^{e}\right)$ and $Y_{m+1}, \ldots, Y_{n}$, we have

$$
\left\langle\left\langle Y_{m+1}\left(q^{e}\right), X\left(t, q^{e}\right)\right\rangle\right\rangle=\cdots=\left\langle\left\langle Y_{n}\left(q^{e}\right), X\left(t, q^{e}\right)\right\rangle\right\rangle=0,
$$

where $\langle\langle\cdot, \cdot\rangle\rangle$ is the inner product operator of two vector fields. Therefore, since (3.9) means $n-m$ equations for the point $q^{e}$, it can be confirmed that the dimension of a neighborhood of $q^{e}: V_{b}^{m}$ is greater than or equal to $m$ from the implicit function theorem $[39,40]$. Consequently, the proof of this lemma is completed.

Now, we derive the main results on a foliation structure of the configuration manifold $Q$ for the completely integrable $A$-rheonomous affine constraints. 
Theorem 3.3. Assume that the A-rheonomous affine constraints (2.2) are completely integrable. Then, the following statements (a) and (b) hold for a time interval $I \subset \mathbf{R}$.

(a) For a neighborhood of a rheonomous regular point $q^{0} \in U_{a}^{m}: V_{a}^{m} \subset U_{a}^{m}$, there exist a local coordinate $\left(U ; x_{1}, \ldots, x_{n}\right)$ and an $m$-dimensional time-varying foliation structure in $V_{a}^{m}$ which is constructed by an m-dimensional time-varying integral manifold determined by the time variable $t$ and the point $q^{0}$ :

$S^{m}\left(t, q^{0}\right):=\left\{q \in V_{a}^{m} \mid x_{m+1}(q)=x_{m+1}\left(\phi_{t}^{X}\left(q^{0}\right)\right), \ldots, x_{n}(q)=x_{n}\left(\phi_{t}^{X}\left(q^{0}\right)\right)\right\}$,

where $\phi_{t}^{X}: Q \rightarrow Q$ is a flow (one-parameter local transformation group) of X. In addition,

$$
\begin{gathered}
C_{0}(t, q)=D(q), \quad C(t, q)=D(q)+\operatorname{span}\{X\}(t, q), \quad \forall q \in U_{a}^{m}, \forall t \in I, \\
\operatorname{dim} C_{0}(t, q)=m, \quad \operatorname{dim} C(t, q)=m+1, \quad \forall q \in U_{a}^{m}, \forall t \in I,
\end{gathered}
$$

hold.

(b) For a neighborhood of a rheonomous equilibrium point $q^{0} \in U_{a}^{m}: V_{b}^{m} \subset U_{b}^{m}$, there exist a local coordinate $\left(U ; x_{1}, \ldots, x_{n}\right)$ and an m-dimensional time-invariant foliation structure in $V_{b}^{m}$ which is constructed by an m-dimensional time-invariant integral manifold determined by the point $q^{0}$ :

$$
S^{m}\left(q^{0}\right):=\left\{q \in V_{b}^{m} \mid x_{m+1}(q)=x_{m+1}\left(q^{0}\right), \ldots, x_{n}(q)=x_{n}\left(q^{0}\right)\right\}
$$

In addition,

$$
\begin{gathered}
C_{0}(t, q)=C(t, q)=D(q), \quad \forall q \in U_{b}^{m}, \quad \forall t \in I, \\
\operatorname{dim} C_{0}(t, q)=\operatorname{dim} C(t, q)=\operatorname{dim} D(q), \quad \forall q \in U_{b}^{m}, \quad \forall t \in I,
\end{gathered}
$$

hold.

Proof. First, we prove the statement (a). See also the illustration depicted in Figure 4 and the proof of Theorem 3.1 in the first paper [38] for understanding the proof. Let us denote the $(n+1)$-dimensional expanded configuration manifold which is constructed by the product manifold of the space of the time variable $\mathbf{R}$ and the configuration manifold $Q$ by $\bar{Q}:=\mathbf{R} \times Q$ with the coordinate $\bar{q}:=\left[t q^{\top}\right]^{\top}$. The $A$-rheonomous affine constraints (2.2) defined on $Q$ can be represented by an expanded Pfaffian equation on $\bar{Q}$ :

$$
\bar{\Omega}(\bar{q})=0
$$

with $n-m$ expanded differential forms:

$$
\bar{\Omega}(\bar{q}):=A(t, q) d t+B(q) d q
$$




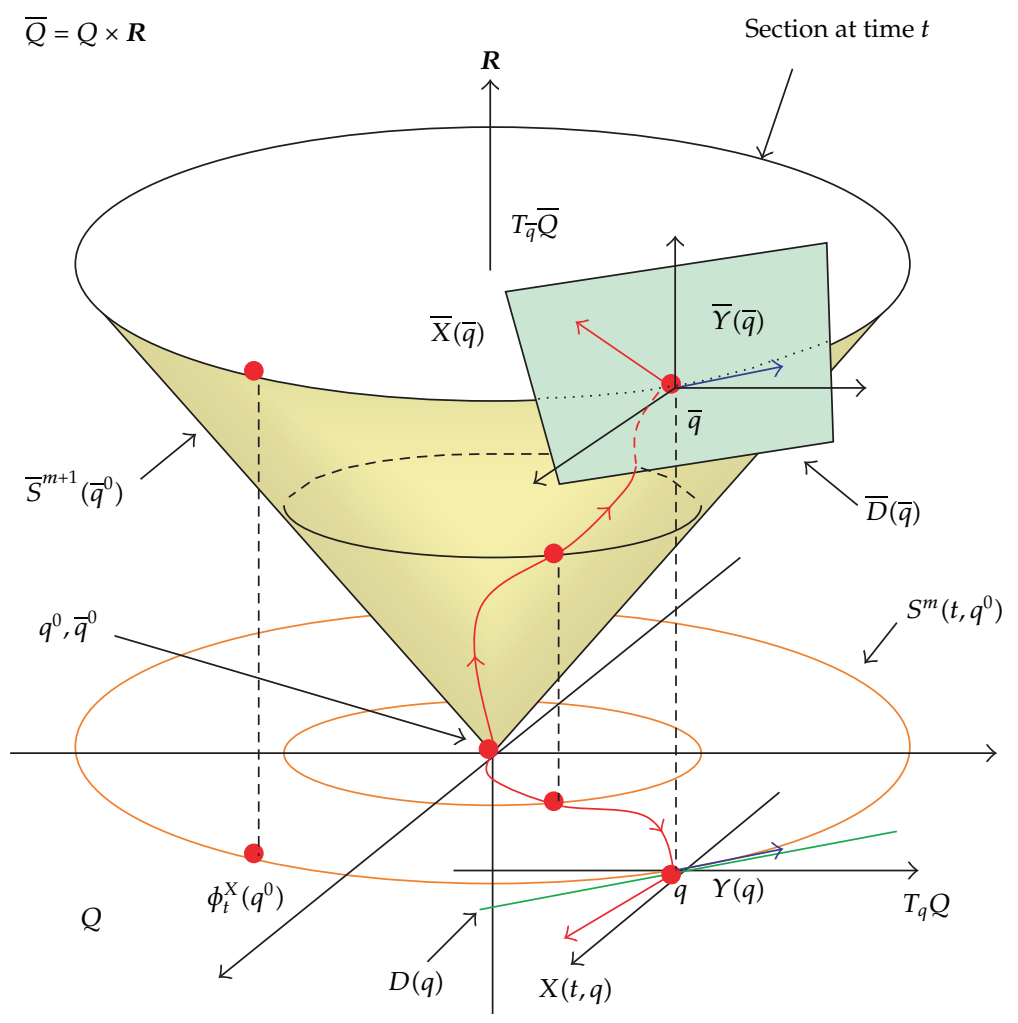

Figure 4: An illustration of $Q$ and $\bar{Q}(n=2, m=1)$.

We can see that $m+1$ annihilators of the $n-m$ expanded differential forms (3.17) as

$$
\bar{X}=\frac{\partial}{\partial t} \oplus X, \quad \bar{Y}_{i}=0 \oplus Y_{i}, \quad i=1, \ldots m .
$$

We also consider a diffeomorphism which is a projection from $\bar{Q}$ to $Q$ at the time $t: \pi_{t}:(t, q) \mapsto$ $q(t)$, and its derivation: $d \pi_{t}: T_{\bar{q}(t)} \bar{Q} \rightarrow T_{q} Q$. Since $d \pi_{t}(\bar{X}(\bar{q}))=X(q)$ holds from the definition of $\bar{X}$ in(3.18), we obtain $\pi_{t}\left(\bar{\phi}_{t}^{\bar{X}}\left(\bar{q}^{0}\right)\right)=\phi_{t}^{X}\left(q^{0}\right)$. In addition, from $\bar{\phi}_{t}^{\bar{X}}\left(\bar{q}^{0}\right) \in \bar{S}^{m+1}\left(\bar{q}^{0}\right)$, it turns out that $\phi_{t}^{\mathrm{X}}\left(q^{0}\right) \in S^{m}\left(t, q^{0}\right)$. Thus, by using a local coordinate $\left(U ; x_{1}, \ldots, x_{n}\right)$, we can represent the $m$-dimensional time-varying integral manifold for the $A$-rheonomous affine constraints (2.2): $S^{m}\left(t, q^{0}\right)$ as (3.10). Furthermore, from the definitions of $C_{0}$ and $C$, it can be confirmed that (3.11) and (3.12) holds.

Next, we consider the proof of the statement (b). Since $d \pi_{t}(\bar{X}(\bar{q}))=X(q) \in D(q)$ holds for a rheonomous affine equilibrium point $q \in V_{b}^{m}$, we can show $\phi_{t}^{X}\left(q^{0}\right) \in S^{m}\left(q^{0}\right)$ by setting $\pi_{0}\left(\bar{S}^{m+1}\left(\bar{q}^{0}\right)\right)=: S^{m}\left(q^{0}\right)$. Hence, $\pi_{t}\left(\bar{S}^{m+1}\left(\bar{q}^{0}\right)\right)=S^{m}\left(q^{0}\right)$ holds and this yields $q^{0} \in S^{m}\left(q^{0}\right)$. Since the dimension of $V_{b}^{m}$ is greater than or equal to $m$ from Lemma 3.2, the $m$-dimensional time-invariant integral manifold for the $A$-rheonomous affine constraints (2.2): $S^{m}\left(q^{0}\right)$ can be represented as (3.13). Moreover, $X(t, q) \in D(q)$ for all $t \in I$ holds in this case, and hence we obtain (3.14) and (3.15) from (3.11) and (3.12), respectively. 


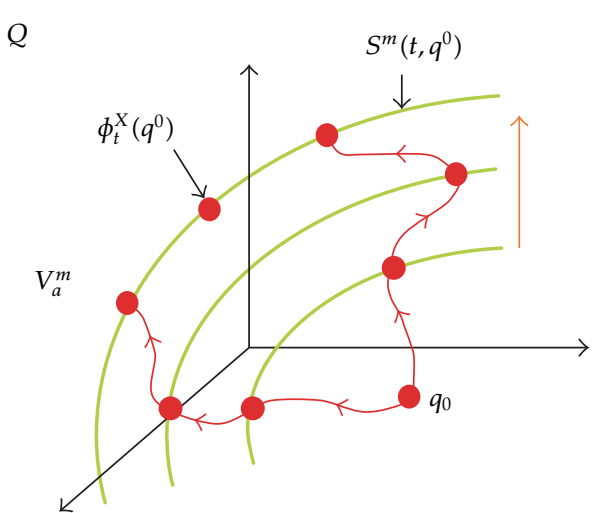

(a) Foliation structure

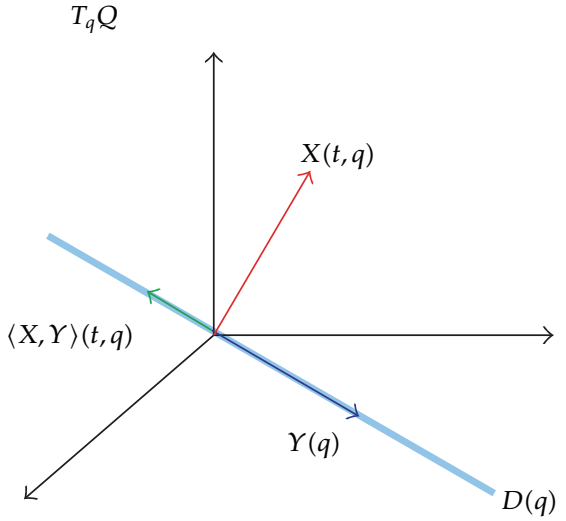

(b) Tangent space

Figure 5: An illustration for Theorem 3.3(a) $(n=3, m=1)$.

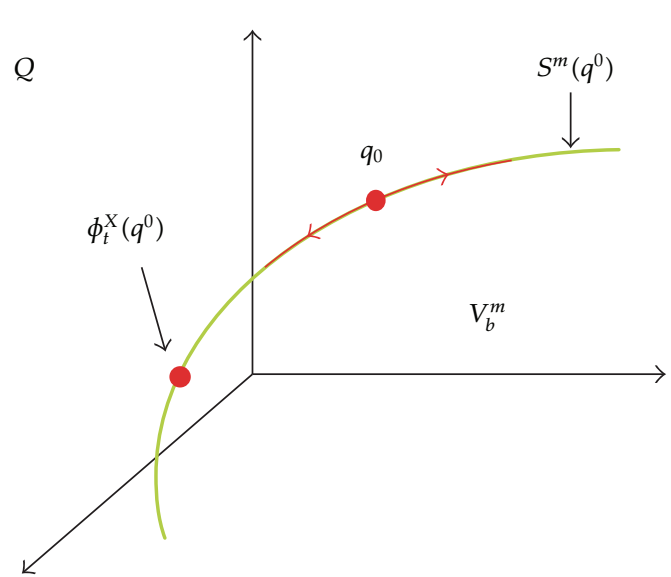

(a) Foliation structure

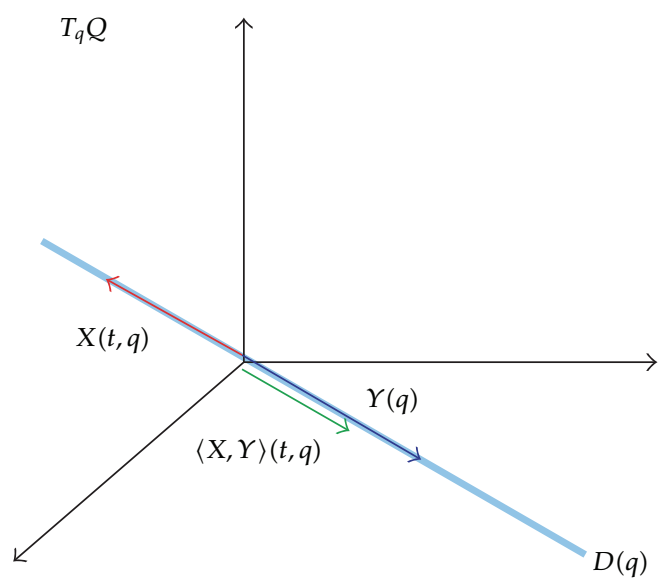

(b) Tangent space

Figure 6: An illustration for Theorem 3.3(b) $(n=3, m=1)$.

The illustrations for Theorem 3.3(a) and (b) are shown in Figures 5 and 6 for the case of $n=3, m=1$, respectively. As shown in Figure 5(a), $V_{a}^{m}$ is divided into a 1-dimensional time-varying foliation structure, and a trajectory starting from an initial point $q_{0}$ at the time $t$ is constrained in the foliation $S^{m}\left(t, q^{0}\right)$. However, since the foliation $S^{m}\left(t, q^{0}\right)$ slides as time goes by, the trajectory can move as a 2-dimensional behavior in $V_{a}^{m}$. In addition, just like the illustration of the tangent space $T_{q} Q$ shown in Figure $5(\mathrm{~b}),\langle X, Y\rangle(t, q)$ lies on $D(q)$, however, $X(t, q)$ does not belong to $D(q)$. This fact also causes the time-varying foliation structure.

On the other hand, $V_{b}^{m}$ is divided into a 1-dimensional time-invariant foliation structure as shown in Figure 6(a). Hence, a trajectory that starts from an initial point $q_{0}$ at the time $t$ is constrained in the foliation $S^{m}\left(q^{0}\right)$ and cannot move to another foliation. Moreover, as the illustration of the tangent space $T_{q} Q$ shown in Figure 6(b), not only $\langle X, Y\rangle(t, q)$ but also $X(t, q)$ lies on $D(q)$, and hence the time-invariant foliation structure occurs.

From the statement (a) in Theorem 3.3, we can confirm the existence of time-varying foliation structures in configuration manifolds for the completely integrable $A$-rheonomous 
affine constraints as is the case in the scleronomous affine constraints [23, 24]. Now, let us consider a simple example in order for graphic understanding of Theorem 3.3 as follows.

Example 3.4. We deal with the same example which is shown in the first paper [38, Example 1]. Consider a configuration manifold:

$$
Q=\left\{q=\left[\begin{array}{l}
q_{1} \\
q_{2} \\
q_{3}
\end{array}\right] \in \mathbf{R}^{3} \mid q_{2}>0, q_{3}>0\right\}
$$

with $n=3$, and $A$-rheonomous affine constraints on $Q$ :

$$
\underbrace{\left[\begin{array}{c}
t q_{1} q_{2} q_{3} \\
0
\end{array}\right]}_{A(t, q)}+\underbrace{\left[\begin{array}{ccc}
q_{2} q_{3} & q_{1} q_{3} & q_{1} q_{2} \\
0 & 1 & 0
\end{array}\right]}_{B(q)}\left[\begin{array}{c}
\dot{q}_{1} \\
\dot{q}_{2} \\
\dot{q}_{3}
\end{array}\right]=0
$$

with $m=1$. It turns out that Assumption 2.1 holds for (3.20). One of the geometric representation for (3.20) can be obtained as follows:

$$
X(t, q)=\left[\begin{array}{c}
-t q_{1} \\
0 \\
0
\end{array}\right], \quad Y(q)=\left[\begin{array}{c}
-q_{1} \\
0 \\
q_{3}
\end{array}\right]
$$

Calculating an iterated rheonomous brackets for $X$ and $Y$ above, we obtain

$$
\langle X, Y\rangle=\frac{\partial Y}{\partial t}+[X, Y]=0+\frac{\partial Y}{\partial q} X-\frac{\partial X}{\partial q} Y=\left[\begin{array}{c}
t q_{1}-t q_{1} \\
0 \\
0
\end{array}\right]=0
$$

Hence, it turns out that all the iterated rheonomous bracket of $X, Y$ are 0 . Therefore, we have

$$
C_{0}=\operatorname{span}\{Y\},
$$

and it turns out that

$$
\operatorname{dim} C_{0}=1, \quad \forall q \in Q, t \in \mathbf{R},
$$

holds. From Theorem 3.1, we can see that the A-rheonomous affine constraints (3.20) are completely integrable. By using the integrating algorithm introduced in Section 4, we can 


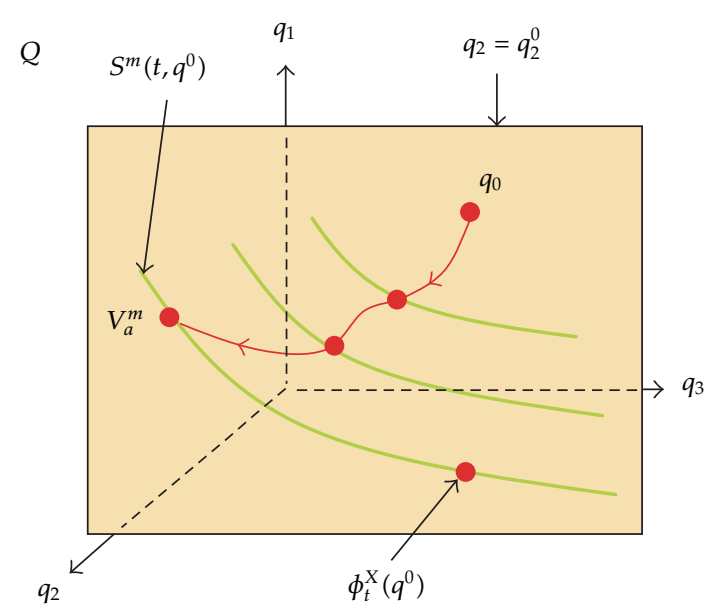

(a) $V_{a}^{m}$

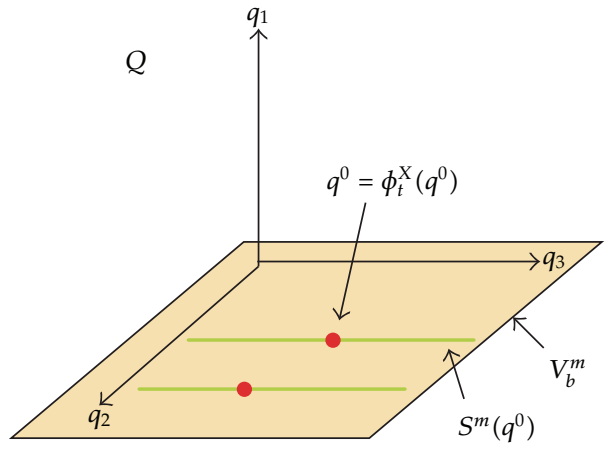

(b) $V_{b}^{m}$

Figure 7: Foliation structures of Example 3.4.

calculate two independent first integrals of (3.20) as

$$
h_{1}(t, q)=q_{2}-q_{2}^{0}, \quad h_{2}(t, q)=\frac{q_{1} q_{3} e^{(1 / 2) t^{2}}}{q_{3}^{0}}-q_{1}^{0},
$$

where $q^{0}=\left[q_{1}^{0} q_{2}^{0} q_{3}^{0}\right]^{\top} \in Q$ is an initial point at the initial time (for the derivation of it, see Example 4.3 in Section 4).

From Theorem 3.3, the set of the rheonomous affine regular points is given by $U_{a}^{m}=$ $V_{a}^{m}=\left\{q \in Q \mid t>0, q_{1} \neq 0\right\}$, and the set of the rheonomous affine regular points is represented by $U_{b}^{m}=V_{b}^{m}=\left\{q \in Q \mid t>0, q_{1}=0\right\}$. That is to say, the $q_{2}-q_{3}$ plane is coincident with $V_{b}^{m}$ and the total space of $Q$ except the $q_{2}-q_{3}$ plane is $V_{a}^{m}$. The illustrations of the foliation structures for these cases are depicted in Figure 7. As shown in Figure 7(a), a trajectory starting from a rheonomous affine regular point $q^{0} \in V_{a}^{m}$ is included in the 1-dimensional time-varying integral manifold $S^{m}\left(t, q^{0}\right)$ at the time $t$, and moves in the 2-dimensional space determined by $q_{2}=q_{2}^{0}$. Therefore, $V_{a}^{m}$ has a 1-dimensional time-varying foliation structure. Meanwhile, as shown in Figure 7(b), a trajectory starting from a rheonomous affine equilibrium point $q^{0} \in V_{b}^{m}$ is included in the 1-dimensional time-invariant integral manifold $S^{m}\left(q^{0}\right)$ and moves only in $S^{m}\left(q^{0}\right)$. Consequently, $V_{b}^{m}$ has a 1-dimensional time-invariant foliation structure.

\subsection{Partially Integrable Case}

Next, we investigate foliation structures of a configuration manifold for the partially integrable $A$-rheonomous affine constraints case in this subsection. Now, necessary and sufficient conditions on partial integrability for the $A$-rheonomous affine constraints (2.2), which are also originally derived in the first paper [38], are shown as the follows.

Theorem 3.5 (see [38]). For the A-rheonomous affine constraints (2.2) defined on an $n$-dimensional manifold $Q$ and a time interval $I \subset \mathbf{R}$, the following statements (a)-(c) are equivalent to each other. 
If they hold, the A-rheonomous affine constraints (2.2) are said to be kth-order partially integrable or $k$ th-order partially holonomic, where $m<k<n$.

(a) There exist $n-k$ independent first integrals of the A-rheonomous affine constraints (2.2): $h_{1}(t, q), \ldots, h_{n-k}(t, q)$ such that

$$
\begin{gathered}
\frac{\partial h_{i}(t, q)}{\partial t}+X(t, q) h_{i}(t, q)=0, \quad i=1, \ldots, n-k, \forall q \in Q, \forall t \in I, \\
Z(t, q) h_{i}(t, q)=0, \quad \forall Z \in C_{0}, i=1, \ldots, n-k, \forall q \in Q, \forall t \in I, \\
d h_{1} \wedge \cdots \wedge d h_{n-k}(t, q) \neq 0, \quad \forall q \in Q, \quad \forall t \in I,
\end{gathered}
$$

hold

(b) There exists a $k$-dimensional time-varying integral manifold $S^{k}\left(t, q^{0}\right)$ of the A-rheonomous affine constraints (2.2) determined by an initial point $q^{0} \in Q$ such that

$$
T_{q} S^{k}\left(t, q^{0}\right)=C_{0}(t, q), \quad \forall q \in S^{k}\left(t, q^{0}\right), \forall t \in I,
$$

holds.

(c) For a smallest and involutive time-varying distribution $C_{0}$,

$$
\operatorname{dim} C_{0}(t, q)=k, \quad \forall q \in Q, \forall t \in I,
$$

holds.

From the statement $(\mathrm{b})$ in Theorem 3.5, it turns out that there exists a $k(m<k<n)$ dimensional time-varying integral manifold in the $n$-dimensional configuration manifold $Q$. Similar to the results in the previous section, we can expect that this time-varying integral manifold creates a foliation structure in $Q$. So, we investigate the foliation structure with much circumstance. We now derive the next lemma that helps to prove the main theorem.

Lemma 3.6. Assume that the A-rheonomous affine constraints (2.2) are kth-order completely integrable. One calls a point $q \in Q$ such that $X(t, q) \notin C_{0}(t, q)$, for all $t \in I$ a kth order rheonomous affine regular point, and denotes a set of $k$ th-order rheonomous affine regular points by

$$
U_{a}^{k}:=\left\{q \in Q \mid X(t, q) \notin C_{0}(t, q), \forall t \in I\right\},
$$

Where $I \subset \mathbf{R}$ is a time interval. On the other hand, one call a point $q \in Q$ such that $X(t, q) \in$ $C_{0}(t, q)$, for all $t \in I$ a kth-order rheonomous affine equilibrium point, and denotes a set of kth-order rheonomous affine equilibrium points by

$$
U_{b}^{k}:=\left\{q \in Q \mid X(t, q) \notin C_{0}(t, q) \forall t \in I\right\} .
$$


Then, the following statements $(a)$ and $(b)$ hold.

(a) For $U_{a}^{k}$ and $U_{b}^{k}$,

$$
U_{a}^{k} \cap U_{b}^{k}=\phi, \quad U_{a}^{k} \cup U_{b}^{k}=Q
$$

hold.

(b) For a neighborhood $V_{b}^{k} \subset U_{b}^{k}$ of a rheonomous affine equilibrium point $q^{e} \in U_{b}^{m}$,

$$
\operatorname{dim} V_{b}^{k} \geq k
$$

holds.

Proof. Similar to the proof of Lemma 3.2, since each point in $Q$ is classified into either a $k$ thorder rheonomous affine regular point or a $k$ th-order rheonomous affine equilibrium point from the definition of these points, the statement (a) obviously holds. Now, consider vector fields $Y_{m+1}, \ldots, Y_{k}$ which are independent of $Y_{1}, \ldots, Y_{m}$ and satisfy

$$
C_{0}=\operatorname{span}\left\{Y_{1}, \ldots, Y_{m}, Y_{m+1}, \ldots, Y_{k}\right\}
$$

Since a $k$ th-order rheonomous affine equilibrium point $q^{e} \in U_{b}^{m}$ satisfies $X\left(q^{e}\right) \in C_{0}\left(q^{e}\right)$, $X\left(t, q^{e}\right)$ can be represented as

$$
X\left(t, q^{e}\right)=\beta_{1}\left(t, q^{e}\right) Y_{1}\left(q^{e}\right)+\cdots+\beta_{k}\left(t, q^{e}\right) Y_{k}\left(q^{e}\right)
$$

with $k$ functions $\beta_{1}(t, q), \ldots, \beta_{k}(t, q)$. We here consider $n-k$ vector fields $Y_{k+1}, \ldots, Y_{n}$ which are independent of each other and are orthogonal to $Y_{1}, \ldots, Y_{k}$. Calculating inner products of $X\left(t, q^{e}\right)$ and $Y_{k+1}, \ldots, Y_{n}$, we have

$$
\left\langle\left\langle Y_{k+1}\left(q^{e}\right), X\left(t, q^{e}\right)\right\rangle\right\rangle=\cdots=\left\langle\left\langle Y_{n}\left(q^{e}\right), X\left(t, q^{e}\right)\right\rangle\right\rangle=0
$$

So, since (3.35) can be considered as $n-k$ equations for the point $q^{e}$, we can see that the dimension of a neighborhood of $q^{e}: V_{b}^{k}$ is greater than or equal to $k$ from the implicit function theorem $[39,40]$. Hence, we have completed the proof.

Hence, we now derive the main results on a foliation structure of the configuration manifold $Q$ for the partially integrable $A$-rheonomous affine constraints as follows.

Theorem 3.7. Assume that the A-rheonomous affine constraints (2.2) are kth order partially integrable. Then, the following statements (a) and (b) hold for a time interval $I \subset \mathbf{R}$.

(a) For a neighborhood of a kth-order rheonomous regular point $q^{0} \in U_{a}^{k}$ : $V_{a}^{k} \subset U_{a}^{k}$, there exist a local coordinate $\left(U ; x_{1}, \ldots, x_{n}\right)$ and a $k$-dimensional time-varying foliation structure in $V_{a}^{k}$ which is constructed by a $k$-dimensional time-varying integral manifold determined by the time variable $t$ and the point $q^{0}$ :

$$
S^{k}\left(t, q^{0}\right):=\left\{q \in V_{a}^{k} \mid x_{k+1}(q)=x_{k+1}\left(\phi_{t}^{X}\left(q^{0}\right)\right), \ldots, x_{n}(q)=x_{n}\left(\phi_{t}^{X}\left(q^{0}\right)\right)\right\} .
$$




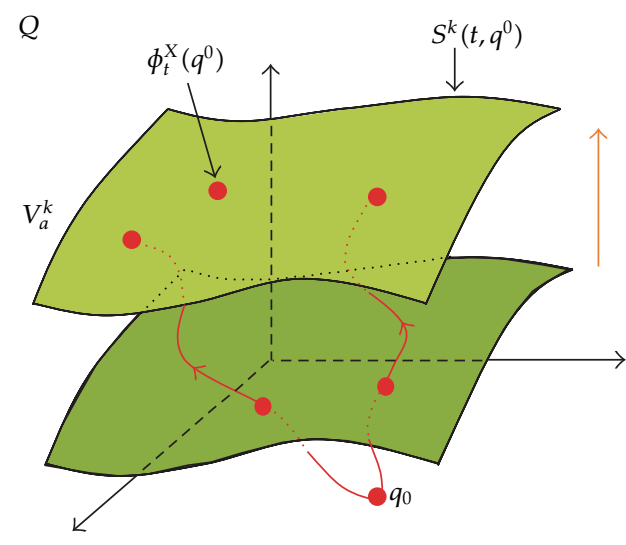

(a) Foliation structure

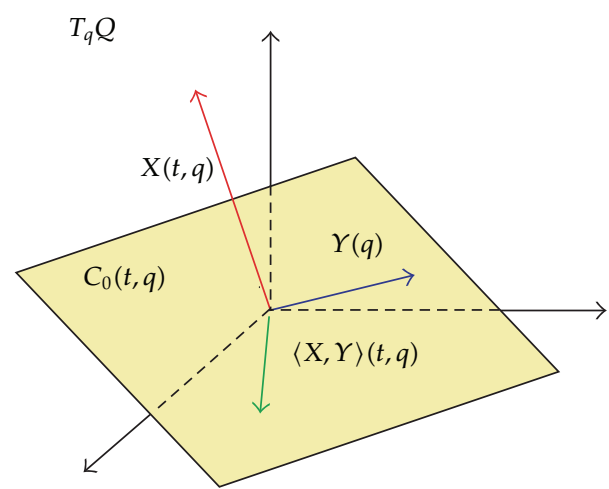

(b) Tangent space

Figure 8: An illustration for Theorem 3.7(a) $(n=3, m=1, k=2)$.

In addition,

$$
\begin{gathered}
C(t, q)=C_{0}(t, q)+\operatorname{span}\{X\}(t, q), \quad \forall q \in U_{a}^{k}, \forall t \in I, \\
\operatorname{dim} C_{0}(t, q)=k, \quad \operatorname{dim} C(t, q)=k+1, \quad \forall q \in U_{a}^{k}, \forall t \in I,
\end{gathered}
$$

hold.

(b) For a neighborhood of a kth-order rheonomous equilibrium point $q^{0} \in U_{a}^{k}: V_{b}^{m} \subset U_{b^{\prime}}^{k}$ there exist a local coordinate $\left(U ; x_{1}, \ldots, x_{n}\right)$ and a $k$-dimensional time-invariant foliation structure in $V_{b}^{k}$ which is constructed by a $k$-dimensional time-invariant integral manifold determined by the point $q^{0}$ :

$$
S^{k}\left(q^{0}\right):=\left\{q \in V_{b}^{m} \mid x_{k+1}(q)=x_{k+1}\left(q^{0}\right), \ldots, x_{n}(q)=x_{n}\left(q^{0}\right)\right\} .
$$

In addition,

$$
\begin{gathered}
C_{0}(t, q)=C(t, q), \quad \forall q \in U_{b}^{k}, \forall t \in I, \\
\operatorname{dim} C_{0}(t, q)=\operatorname{dim} C(t, q)=k, \quad \forall q \in U_{b}^{k}, \forall t \in I,
\end{gathered}
$$

hold.

Proof. This theorem can be proven by similar procedure to the proof of Theorem 3.1 and the results in Lemma 3.6. The details are omitted.

Figures 8 and 9 show the illustrations for Theorem 3.7(a) and (b) for the case of $n=3$, $m=1, k=2$, respectively. As shown in Figure $8(\mathrm{a}), V_{a}^{k}$ is divided into a 2-dimensional timevarying foliation structure, and a trajectory that starts from an initial point $q_{0}$ at the time $t$ is included in the foliation $S^{k}\left(t, q^{0}\right)$. However, since the foliation $S^{k}\left(t, q^{0}\right)$ slides as time goes by, the trajectory can move as a 3-dimensional behavior in $V_{a}^{m}$. In addition, like the illustration of 


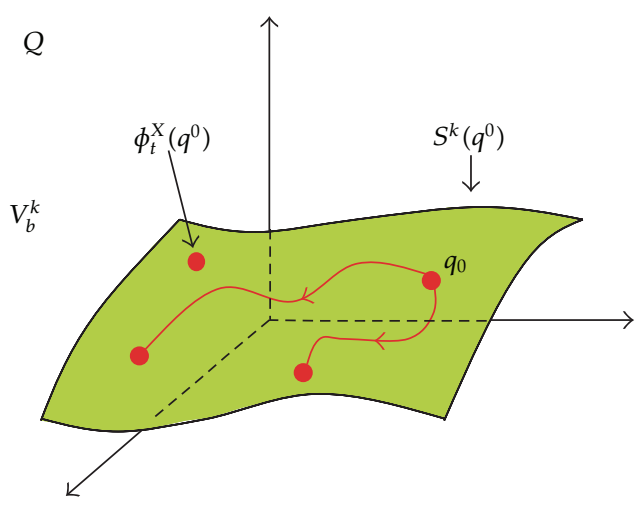

(a) Foliation structure

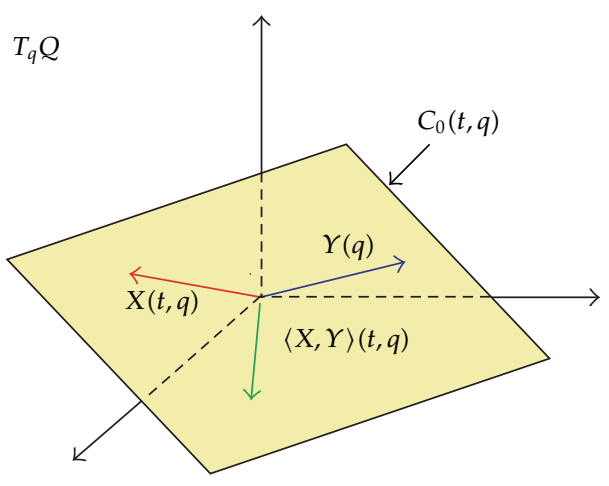

(b) Tangent space

Figure 9: An illustration for Theorem 3.7(b) $(n=3, m=1, k=2)$.

the tangent space $T_{q} Q$ shown in Figure 8(b), $\langle X, Y\rangle(t, q)$ lies on $C(t, q)$, however, $X(t, q)$ does not belong to $C(t, q)$. This result also causes the time-varying foliation structure.

In contrast, $V_{b}^{k}$ is divided into a 2-dimensional time-invariant foliation structure as shown in Figure 9(a), Therefore, a trajectory starting from an initial point $q_{0}$ at the time $t$ is constrained in the foliation $S^{m}\left(q^{0}\right)$ and cannot move to another foliation. Moreover, as the illustration of the tangent space $T_{q} Q$ shown in Figure $9(b), X(t, q)$ as well as $\langle X, Y\rangle(t, q)$ lies on $C(t, q)$, and hence we can find the time-invariant foliation structure in this case.

Similar to the statement (a) in Theorem 3.3, from the statement (a) in Theorem 3.7, it can be also confirmed that there exist time-varying foliation structures in configuration manifolds for the partially integrable $A$-rheonomous affine constraints, which are in common with the case of the scleronomous affine constraints [23, 24]. We here consider a simple example in order to understand Theorem 3.7 as follows.

Example 3.8. We here consider the same example which is shown in the first paper [38, Example 2]. Let us consider a 3-dimensional configuration manifold:

$$
Q=\left\{q=\left[\begin{array}{l}
q_{1} \\
q_{2} \\
q_{3}
\end{array}\right] \in \mathbf{R}^{3} \mid q_{2}>0, q_{3}>0\right\}
$$

with $n=3$ and $A$-rheonomous affine constraints on $Q$ :

$$
\underbrace{\left[\begin{array}{c}
t q_{1} q_{2} q_{3} \\
q_{2} q_{3}
\end{array}\right]}_{A(t, q)}+\underbrace{\left[\begin{array}{ccc}
q_{2} q_{3} & q_{1} q_{3} & q_{1} q_{2} \\
0 & 1 & q_{2}
\end{array}\right]}_{B(q)}\left[\begin{array}{c}
\dot{q}_{1} \\
\dot{q}_{2} \\
\dot{q}_{3}
\end{array}\right]=0
$$

with $m=1$. We can see that Assumption 2.1 holds for (3.41). One of the geometric 
representation for (3.41) can be obtained as follows:

$$
X(t, q)=\left[\begin{array}{c}
-t q_{1}+q_{1} q_{3} \\
-q_{2} q_{3} \\
0
\end{array}\right], \quad Y(q)=\left[\begin{array}{c}
-q_{1}+q_{1} q_{3} \\
-q_{2} q_{3} \\
q_{3}
\end{array}\right]
$$

By calculating iterated rheonomous brackets for $X$ and $Y$ above, we have

$$
\begin{gathered}
\langle X, Y\rangle=\frac{\partial Y}{\partial t}+[X, Y]=0+\frac{\partial Y}{\partial q} X-\frac{\partial X}{\partial q} Y=\left[\begin{array}{c}
-q_{1} q_{3} \\
q_{2} q_{3} \\
0
\end{array}\right], \\
\langle X,\langle X, Y\rangle\rangle=\frac{\partial\langle X, Y\rangle}{\partial t}+[X,\langle X, Y\rangle]=\left[\begin{array}{c}
t q_{1} q_{3}-q_{1} q_{3}^{2} \\
-q_{2} q_{3}^{2} \\
0
\end{array}\right]-\left[\begin{array}{c}
t q_{1} q_{3}-q_{1} q_{3}^{2} \\
-q_{2} q_{3}^{2} \\
0
\end{array}\right]=0, \\
\langle Y,\langle X, Y\rangle\rangle=[Y,\langle X, Y\rangle]=\left[\begin{array}{c}
-q_{1} q_{3}^{2} \\
-q_{2} q_{3}^{2}+q_{2} q_{3} \\
0
\end{array}\right]-\left[\begin{array}{c}
q_{1} q_{3}-q_{1} q_{3}^{2} \\
-q_{2} q_{3}^{2} \\
0
\end{array}\right]=\left[\begin{array}{c}
-q_{1} q_{3} \\
q_{2} q_{3} \\
0
\end{array}\right],
\end{gathered}
$$$$
\vdots
$$

Therefore, we have

$$
C_{0}=\operatorname{span}\{Y,\langle X, Y\rangle\}
$$

and we can see that

$$
\operatorname{dim} C_{0}=2, \quad \forall q \in Q, \forall t \in \mathbf{R}
$$

holds. From Theorem 3.3, we can find that the A-rheonomous affine constraints (3.41) are 2nd-order partial nonintegrable. By using the integrating algorithm introduced in Section 4, we can obtain two independent first integrals of (3.41) as

$$
h_{1}(t, q)=\frac{q_{1} q_{2} q_{3} e^{(1 / 2) t^{2}}}{q_{2}^{0} q_{3}^{0}}-q_{1}^{0}
$$

where $q^{0}=\left[q_{1}^{0} q_{2}^{0} q_{3}^{0}\right]^{\top} \in Q$ is an initial point at the initial time (for the derivation of it, see Example 4.6 in Section 4).

From Theorem 3.7, the set of the 2nd-order rheonomous affine regular points is represented by $U_{a}^{k}=V_{a}^{k}=\left\{q \in Q \mid t>0, q_{1} \neq 0\right\}$, and the set of the 2nd-order rheonomous affine regular points is given by $U_{b}^{m}=V_{b}^{m}=\left\{q \in Q \mid t>0, q_{1}=0\right\}$. Hence, the $q_{2}-q_{3}$ plane corresponds to $V_{b}^{k}$ and the total space of $Q$ except the $q_{2}-q_{3}$ plane is $V_{a}^{k}$. Figure 10 


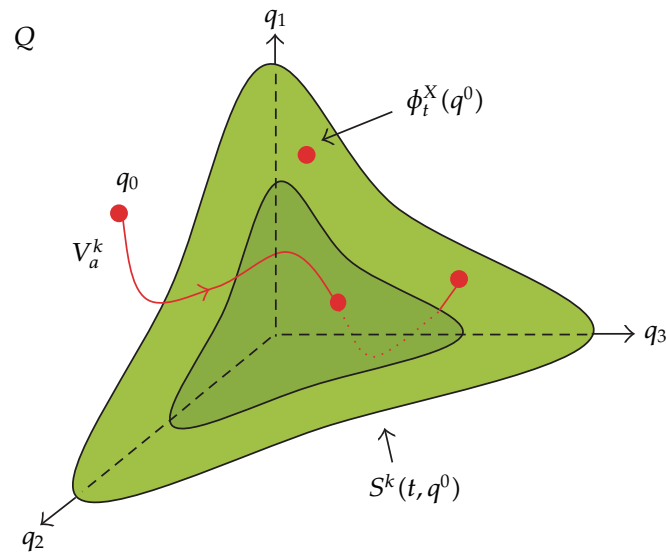

(a) $V_{a}^{k}$

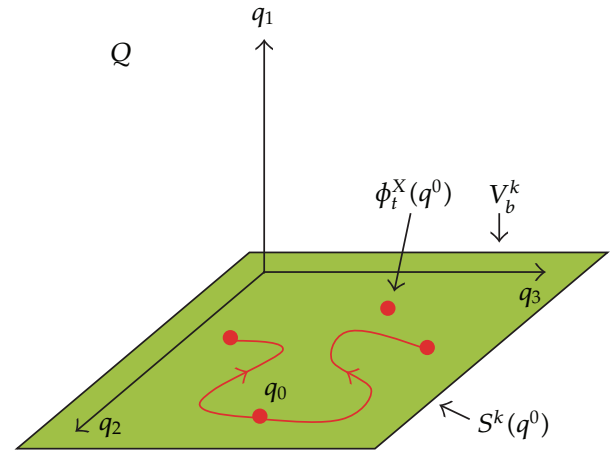

(b) $V_{b}^{k}$

Figure 10: Foliation structures of Example 3.8.

depicts the illustrations of the foliation structures for these cases. As shown in Figure 10(a), a trajectory starting from a 2nd-order rheonomous affine regular point $q^{0} \in V_{a}^{k}$ is included in the 2-dimensional time-varying integral manifold $S^{k}\left(t, q^{0}\right)$ at the time $t$, and it moves in the 3-dimensional space determined by $q_{2}=q_{2}^{0}$. Therefore, $V_{a}^{k}$ has a 2-dimensional time-varying foliation structure. On the other hand, as shown in Figure 10(b), a trajectory that starts from a 2nd rheonomous affine equilibrium point $q^{0} \in V_{b}^{m}$ is included in the 2-dimensional timeinvariant integral manifold $S^{k}\left(q^{0}\right)$ and moves only in $S^{k}\left(q^{0}\right)$. Consequently, $V_{b}^{k}$ has a 2dimensional time-invariant foliation structure.

\subsection{Completely Nonintegrable Case}

In this subsection, we finally deal with the case where the $A$-rheonomous affine constraints are completely nonintegrable. Now, we present necessary and sufficient conditions on complete nonintegrability for the $A$-rheonomous affine constraints (2.2), which are originally obtained in the first paper [38] as follow.

Theorem 3.9 (see [38]). For the A-rheonomous affine constraints (2.2) defined on an $n$-dimensional manifold $Q$ and a time interval $I \subset \mathbf{R}$, the following statements $(a),(b)$ are equivalent to each other. If they hold, the A-rheonomous affine constraints (2.2) are said to be completely nonintegrable or completely nonholonomic.

(a) There do not exist independent first integrals of the A-rheonomous affine constraints (2.2).

(b) For a smallest and involutive time-varying distribution $C_{0}$,

$$
\operatorname{dim} C_{0}(t, q)=n, \quad \forall q \in Q, \forall t \in I,
$$

holds.

From the statement (a) in Theorem 3.9, it can be easily expected that there exist no foliation structure in $Q$. We summarize this fact as the following theorem. 


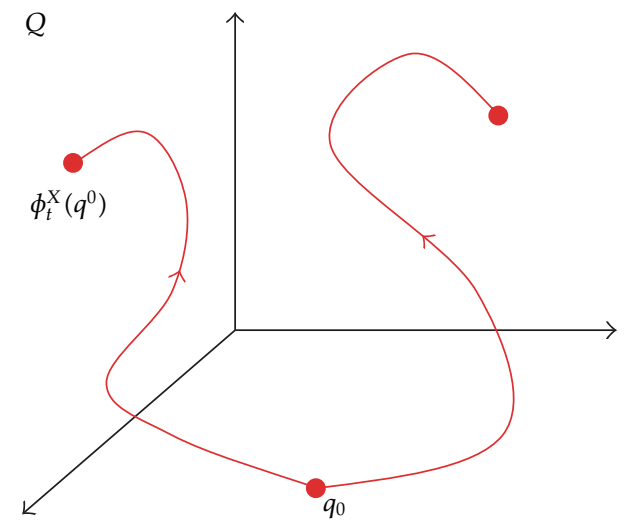

(a) Foliation structure

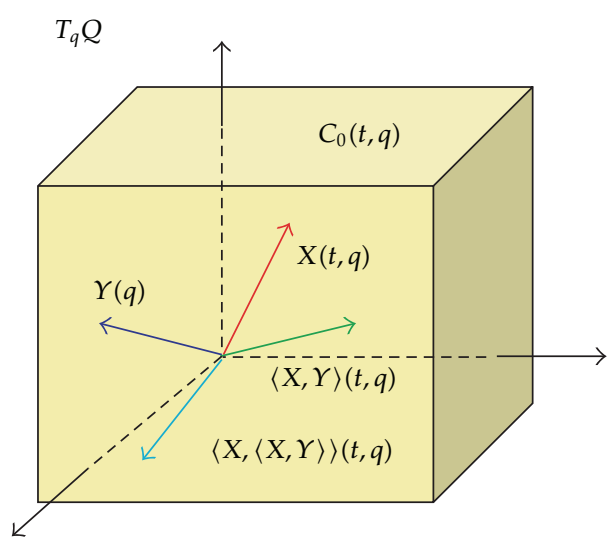

(b) Tangent space

Figure 11: An illustration for Theorem $3.10(n=3, m=1)$.

Theorem 3.10. Assume that the A-rheonomous affine constraints (2.2) are completely nonintegrable. Then, for a time interval $I \subset \mathbf{R}$,

$$
X\left(t, q^{0}\right) \in C_{0}\left(q^{0}\right), \quad q^{0} \in Q, t \in I,
$$

holds and there is no foliation structure in $Q$. In addition,

$$
\begin{gathered}
C_{0}(t, q)=C(t, q)=T_{q} Q, \quad \forall q \in Q, \forall t \in I, \\
\operatorname{dim} C_{0}(t, q)=\operatorname{dim} C(t, q)=n, \quad \forall q \in Q, \forall t \in I,
\end{gathered}
$$

hold.

Proof. We can prove this theorem by considering the case of $k=n$ in the results in Theorem 3.7. The details are omitted.

For Theorem 3.10, the illustration is depicted for the case of $n=3, m=1, k=2$ in Figure 11. As shown in Figure 11(a), a trajectory starting from an initial point $q^{0} \in Q$ can move in $Q$ without constraints by integral manifolds. Therefore, $Q$ is not divided into a foliation structure. In addition, as the illustration of the tangent space $T_{q} Q$ shown in Figure 11(b), neither $\langle X, Y\rangle(t, q)$ nor $\langle X,\langle X, Y\rangle\rangle(t, q)$ do not belong to $D(q)$ and three vector fields $Y(q),\langle X, Y\rangle(t, q),\langle X,\langle X, Y\rangle\rangle(t, q)$ span a 3-dimensional space. This result also causes no foliation structure in $Q$. as follows.

Now, let us deal with a simple example in order to verify the results in Theorem 3.10 
Example 3.11. Now, we treat the same example which is shown in the first paper [38, Example 3]. Consider a 3-dimensional configuration manifold:

$$
Q=\left\{q=\left[\begin{array}{l}
q_{1} \\
q_{2} \\
q_{3}
\end{array}\right] \in \mathbf{R}^{3}\right\}
$$

with $n=3$, and $A$-rheonomous affine constraints defined on $Q$ :

$$
\underbrace{\left[\begin{array}{c}
t q_{2} \\
-q_{1}
\end{array}\right]}_{A(t, q)}+\underbrace{\left[\begin{array}{ccc}
1 & 0 & -1 \\
0 & 1 & 1
\end{array}\right]}_{B(q)}\left[\begin{array}{l}
\dot{q}_{1} \\
\dot{q}_{2} \\
\dot{q}_{3}
\end{array}\right]=0
$$

with $m=1$. We can see that Assumption 2.1 holds for (3.51). One of the geometric representation for (3.51) can be obtained:

$$
X(t, q)=\left[\begin{array}{c}
-t q_{2} \\
q_{1} \\
0
\end{array}\right], \quad Y(q)=\left[\begin{array}{c}
1 \\
-1 \\
1
\end{array}\right]
$$

Calculating iterated rheonomous brackets for $X$ and $Y$ above, we have

$$
\begin{gathered}
\langle X, Y\rangle=\frac{\partial Y}{\partial t}+[X, Y]=0+\frac{\partial Y}{\partial q} X-\frac{\partial X}{\partial q} Y=\left[\begin{array}{c}
-t \\
0 \\
0
\end{array}\right] \\
\langle X,\langle X, Y\rangle\rangle=\frac{\partial\langle X, Y\rangle}{\partial t}+[X,\langle X, Y\rangle]=\left[\begin{array}{c}
-1 \\
0 \\
0
\end{array}\right]+\left[\begin{array}{l}
0 \\
t \\
0
\end{array}\right]=\left[\begin{array}{c}
-1 \\
t \\
0
\end{array}\right], \\
\langle\langle X, Y\rangle, X\rangle=-\frac{\partial\langle X, Y\rangle}{\partial t}+[\langle X, Y\rangle, X]=\left[\begin{array}{l}
1 \\
t \\
0
\end{array}\right] \\
\langle Y,\langle X, Y\rangle\rangle=[Y,\langle X, Y\rangle]=0 \\
\langle\langle X, Y\rangle, Y\rangle=[\langle X, Y\rangle, Y]=0
\end{gathered}
$$

Therefore, we have

$$
C_{0}=\operatorname{span}\{Y,\langle X, Y\rangle,\langle X,\langle X, Y\rangle\rangle\}
$$


and it can be confirmed that

$$
\operatorname{dim} C_{0}=3, \quad \forall q \in Q, \forall t \neq 0
$$

holds. From Theorem 3.9, we can see that the A-rheonomous affine constraints (3.51) are completely nonintegrable. Therefore, from Theorem 3.10, it can be confirmed that there exists no foliation structure in $Q$.

\section{Integrating Algorithm for Integrable $A$-Rheonomous Affine Constraints}

\subsection{Completely Integrable Case}

As seen in the first paper [38] and Section 3 in this second paper, if the A-rheonomous affine constraints (2.2) are completely integrable or partially integrable, there exist some independent first integrals of them, and they form time-varying and time-invariant foliation structures in a configuration manifold $Q$. If we consider reduction of the dimension of a given configuration manifold with the $A$-rheonomous affine constraints, we need the explicit forms of independent first integrals. For scleronomous linear constraints, that is, $B(q) \dot{q}=0$, a method on calculation of independent first integrals of them has been well known $[17,18]$, and for scleronomous affine constraints, that is, $A(q)+B(q) \dot{q}=0$, we have developed algorithms to calculate independent first integrals of them in [25]. However, a method to calculate independent first integrals of given $A$-rheonomous affine constraints has not been proposed. Thus, in this section, we will derive two types of integrating algorithms which can explicitly derive independent first integrals of the $A$-rheonomous affine constraints (2.2).

In this subsection, we first consider the case where the $A$-rheonomous affine constraints (2.2) are completely integrable and derive an integrating algorithm for them. Theorem 3.1 in Section 3 guarantees the existence of $n-m$ independent first integrals of the $A$-rheonomous affine constraints (2.2). Now, we derive an algorithm to calculate the $n-m$ independent first integrals. First of all, we can find $n-m$ vector fields $Y_{m+1}, \ldots, Y_{n}$ such that

$$
\operatorname{span}\left\{Y_{1}, \ldots, Y_{m}, Y_{m+1}, \ldots, Y_{n}\right\}=\mathbf{R}^{n}
$$

holds for the vector fields of the geometric representation for the A-rheonomous affine constraints (2.2): $Y_{1}, \ldots, Y_{m}$. Then, let us denote flows (1-parameter local transformation groups) of $X$ and $Y_{i}$ by $\phi_{t}^{X}$ and $\varphi_{\tau_{i}}^{Y_{i}}$ with parameters $t$ and $\tau_{i}$, respectively. We set an initial point at the initial time $t=0$ as $q^{0} \in Q$. We also consider $m+1$ vector fields defined on the expanded configuration manifold $\bar{Q}=\mathbf{R} \times Q$ (3.18), and then their flows on $Q$ are obtained as

$$
\bar{\phi}_{t}^{\bar{X}}:=\left[\begin{array}{c}
t \\
\phi_{t}^{\mathrm{X}}
\end{array}\right], \quad \bar{\varphi}_{\tau_{i}}^{\bar{Y}_{i}}:=\left[\begin{array}{c}
0 \\
\varphi_{\tau_{i}}^{\gamma_{i}}
\end{array}\right], \quad i=1, \ldots, m .
$$


Calculating the composite mapping of $(n+1)$ flows (4.15) yields

$$
\bar{\Phi}:=\bar{\phi}_{t}^{\bar{X}} \circ \bar{\varphi}_{\tau_{1}}^{\bar{Y}_{1}} \circ \cdots \circ \bar{\varphi}_{\tau_{n}}^{\bar{Y}_{n}}=\left[\begin{array}{c}
t \\
\phi_{t}^{X} \circ \varphi_{\tau_{1}}^{Y_{1}} \circ \cdots \circ \varphi_{\tau_{n}}^{Y_{n}}
\end{array}\right]=\left[\begin{array}{c}
t \\
\Phi_{t}
\end{array}\right],
$$

where

$$
\Phi_{t}:=\phi_{t}^{\mathrm{X}} \circ \varphi_{\tau_{1}}^{Y_{1}} \circ \cdots \circ \varphi_{\tau_{n}}^{Y_{n}}
$$

is the composite mapping of $n+1$ flows $\phi_{t}^{X}, \varphi_{\tau_{i}}^{Y_{i}}$. From (4.16), it turns out that the projection of $\bar{\Phi}$ onto $Q$ is equivalent to $\Phi_{t}$. Hence, by applying the idea of the integrating algorithm for scleronomous linear constraints defined on $Q[17,18]$ to $\mathbf{R} \times Q$ and considering projection of it onto $Q$, we can derive the following algorithm to calculate $n-m$ independent first integrals of completely integrable $A$-rheonomous affine constraints.

Algorithm 4.1. For the case where the A-rheonomous affine constraints (2.2) are completely integrable, we can obtain $n-m$ independent first integrals of them by the following procedure.

Step 1. Set $m+1$ vector fields $X, Y_{1}, \ldots, Y_{m}$ of geometric representation for the $A$-rheonomous affine constraints (2.2).

Step 2. For $X, Y_{1}, \ldots, Y_{m}$, derive linearly independent vector fields $Y_{m+1}, \ldots, Y_{n}$ that satisfy (4.14).

Step 3. Calculate flows of $X, Y_{1}, \ldots, Y_{n}: \phi_{t}^{X}, \varphi_{\tau_{i}}^{Y_{1}}, \ldots, \varphi_{\tau_{i}}^{Y_{n}}$.

Step 4. Combine $n+1$ flows $\phi_{t}^{X}, \varphi_{\tau_{i}}^{Y_{1}}, \ldots, \varphi_{\tau_{i}}^{Y_{n}}$ derived in Step 4 as (4.17).

Step 5. Set $q=\Phi_{t}(\tau)$ and calculate the inverse function $\tau=\Phi_{t}^{-1}(q)$, where $\tau=\left[\begin{array}{lll}\tau_{1} & \cdots & \tau_{n}\end{array}\right]^{\top} \in$ $\mathbf{R}^{n}$. Then, the last $n-m$ components of $\Phi_{t}^{-1}$ are independent first integrals of (2.2).

It must be noted that Algorithm 4.1 is similar to the one for the scleronomous affine constraints case [25] despite the explicit existence of the time variable in the $A$-rheonomous affine constraints (2.2). Moreover, the existence of the inverse mapping $\Phi_{t}^{-1}$ which appears at Step 5 in Algorithm 4.1 is guaranteed by the following proposition.

Proposition 4.2. Assume that the A-rheonomous affine constraints (2.2) are completely integrable. Then, there exists a time interval $I \subset \mathbf{R}$ and $\Phi_{t}(\tau)$ is a diffeomorphism at any time $t \in I$. That is to say, there exists its inverse mapping $\Phi_{t}^{-1}$.

Proof. First, we set $\bar{\tau}:=\left[t \tau^{\top}\right]^{\top}$. Calculating the partial differential of (4.16) with the chain rule of differential calculation, we have

$$
\begin{aligned}
\frac{\partial \bar{\Phi}}{\partial t} & =\frac{\partial}{\partial t}\left\{\bar{\phi}_{t}^{\bar{X}} \circ \bar{\varphi}_{\tau_{1}}^{\bar{Y}_{1}} \circ \cdots \circ \bar{\varphi}_{\tau_{n}}^{\bar{Y}_{n}}\right\} \\
& =\bar{X}\left(\bar{\phi}_{t}^{\bar{X}} \circ \bar{\varphi}_{\tau_{1}}^{\bar{Y}_{1}} \circ \cdots \circ \bar{\varphi}_{\tau_{n}} \bar{Y}_{n}\right),
\end{aligned}
$$




$$
\begin{aligned}
\frac{\partial \bar{\Phi}}{\partial \tau_{i}}= & \frac{\partial \bar{\phi}}{\partial \bar{\tau}} \frac{\partial \bar{\varphi}_{\tau_{1}}}{\partial \bar{\tau}} \cdots \frac{\partial \bar{\varphi}_{\tau_{i-1}}}{\partial \bar{\tau}} \frac{\partial}{\partial \tau_{i}}\left(\bar{\varphi}_{\tau_{i}} \bar{Y}_{i} \circ \cdots \circ \bar{\varphi}_{\tau_{n}} \bar{Y}_{n}\right) \\
& =\frac{\partial \bar{\phi}}{\partial \bar{\tau}} \frac{\partial \bar{\varphi}_{\tau_{1}}}{\partial \bar{\tau}} \cdots \frac{\partial \bar{\varphi}_{\tau_{i-1}}}{\partial \bar{\tau}} \bar{Y}_{i}\left(\bar{\varphi}_{\tau_{i}} \bar{Y}_{i} \circ \cdots \circ \bar{\varphi}_{\tau_{n}}\right) .
\end{aligned}
$$

Substituting $\bar{\tau}=0$ into (4.5), we obtain

$$
\left.\frac{\partial \bar{\Phi}}{\partial t}\right|_{\bar{\tau}=0}=\bar{X}\left(q^{0}\right),\left.\quad \frac{\partial \bar{\Phi}}{\partial \tau_{i}}\right|_{\bar{\tau}=0}=\bar{Y}_{i}\left(q^{0}\right),
$$

that is to say,

$$
\left.\frac{\partial \bar{\Phi}}{\partial t}\right|_{\bar{\tau}=0}=\left[\bar{X}\left(q^{0}\right) \bar{Y}_{1}\left(q^{0}\right) \cdots \bar{Y}_{n}\left(q^{0}\right)\right] .
$$

Since $\bar{X}, \bar{Y}_{1}, \ldots, \bar{Y}_{n}$ are linearly independent of each other,

$$
\left.\operatorname{rank} \frac{\partial \bar{\Phi}}{\partial \bar{\tau}}\right|_{\bar{\tau}=0}=n+1
$$

holds. Hence it can be confirmed that $\bar{\Phi}(\bar{\tau})$ is a diffeomorphism by the implicit function theorem. Since the projection of $\bar{\Phi}(\bar{\tau})$ onto $Q$ is equivalent to $\Phi_{t}(\tau), \Phi_{t}(\tau)$ is also a diffeomorphism. Consequently, the proof of the proposition is completed.

Now, we deal with a simple example in order to check the effectiveness of Algorithm 4.1 and Proposition 2.7 as follows.

Example 4.3. We here consider the A-rheonomous affine constraints (3.50) on the 3dimensional configuration manifold (3.20), which are the same ones in Example 3.4. As seen in Example 3.4, we can confirm that (3.50) are completely integrable with $m=1$, and hence there exist two independent first integrals of (3.50). So, let us calculate them by using Algorithm 4.1. One of the geometric representation for (3.50) is given by (3.21) and we here set $Y_{1}:=Y$. We can easily find two vector fields $Y_{2}, Y_{3}$ satisfying (4.14) as

$$
Y_{2}=\left[\begin{array}{l}
0 \\
1 \\
0
\end{array}\right], \quad Y_{3}=\left[\begin{array}{l}
1 \\
0 \\
0
\end{array}\right]
$$


and then flows of $X, Y_{1}, Y_{2}, Y_{3}$ can be obtained as

$$
\begin{array}{cc}
\phi_{t}^{X}=\left[\begin{array}{c}
q_{1}^{0} e^{-(1 / 2) t^{2}} \\
q_{2}^{0} \\
q_{3}^{0}
\end{array}\right], \quad \varphi_{\tau_{1}}^{Y_{1}}=\left[\begin{array}{c}
q_{1}^{0} e^{-\tau_{1}} \\
q_{2}^{0} \\
q_{3}^{0} e^{\tau_{1}}
\end{array}\right], \\
\varphi_{\tau_{2}}^{\gamma_{2}}=\left[\begin{array}{c}
q_{1}^{0} \\
\tau_{2}+q_{2}^{0} \\
q_{3}^{0}
\end{array}\right], & \varphi_{\tau_{3}}^{\gamma_{3}}=\left[\begin{array}{c}
\tau_{3}+q_{1}^{0} \\
q_{2}^{0} \\
q_{3}^{0}
\end{array}\right],
\end{array}
$$

where $q^{0}=\left[\begin{array}{lll}q_{1}^{0} & q_{2}^{0} & q_{3}^{0}\end{array}\right]^{\top} \in Q$ is an initial point of the flows at the initial time. Combining the flows (4.10) as (4.17), we have

$$
\Phi_{t}(\tau)=\phi_{t}^{X} \circ \varphi_{\tau_{1}}^{Y_{1}} \circ \varphi_{\tau_{2}}^{Y_{2}} \circ \varphi_{\tau_{3}}^{Y_{3}}=\left[\begin{array}{c}
\left(\tau_{3}+q_{1}^{0}\right) e^{-\tau_{1}} e^{-(1 / 2) t^{2}} \\
\tau_{2}+q_{2}^{0} \\
q_{3}^{0} e^{\tau_{1}}
\end{array}\right]
$$

and from the equation $q=\Phi_{t}(\tau)$, we can calculate the inverse mapping of $\Phi_{t}$ as follows:

$$
\Phi_{t}^{-1}(q)=\left[\begin{array}{c}
\log \frac{q_{3}^{0}}{q_{3}} \\
q_{2}-q_{2}^{0} \\
\frac{q_{1} q_{3} e^{(1 / 2) t^{2}}}{q_{3}^{0}}-q_{1}^{0}
\end{array}\right]
$$

Consequently, we have two independent first integrals of (3.50):

$$
h_{1}(t, q)=q_{2}-q_{2}^{0}, \quad h_{2}(t, q)=\frac{q_{1} q_{3} e^{(1 / 2) t^{2}}}{q_{3}^{0}}-q_{1}^{0}
$$

as the last two components of (4.12). It can be also confirmed that these two first integrals (4.13) satisfy (3.1) and hence (4.13) are independent of each other.

\subsection{Partially Integrable Case}

As seen in the first paper [38] and Section 3 in this second paper, if the A-rheonomous affine constraints (2.2) are completely integrable or partially integrable, there exist some independent first integrals of them, and they form time-varying and time-invariant foliation structures in a configuration manifold $Q$. If we consider reduction of the dimension of a given configuration manifold with the $A$-rheonomous affine constraints, we need the explicit forms of independent first integrals. For scleronomous linear constraints, that is, $B(q) \dot{q}=0$, a method on calculation of independent first integrals of them has been well known $[17,18]$, 
and for scleronomous affine constraints, that is, $A(q)+B(q) \dot{q}=0$, we have developed algorithms to calculate independent first integrals of them in [25]. However, a method to calculate independent first integrals of given $A$-rheonomous affine constraints has not been proposed. Thus, in this section, we will derive two types of integrating algorithms which can explicitly derive independent first integrals of the $A$-rheonomous affine constraints (2.2).

In this subsection, we first consider the case where the $A$-rheonomous affine constraints (2.2) are completely integrable and derive an integrating algorithm for them. Theorem 3.1 in Section 3 guarantees the existence of $n-m$ independent first integrals of the $A$-rheonomous affine constraints (2.2). Now, we derive an algorithm to calculate the $n-m$ independent first integrals. First of all, we can find $n-m$ vector fields $Y_{m+1}, \ldots, Y_{n}$ such that

$$
\operatorname{span}\left\{Y_{1}, \ldots, Y_{m}, Y_{m+1}, \ldots, Y_{n}\right\}=\mathbf{R}^{n}
$$

holds for the vector fields of the geometric representation for the $A$-rheonomous affine constraints (2.2): $Y_{1}, \ldots, Y_{m}$. Then, let us denote flows (1-parameter local transformation groups) of $X$ and $Y_{i}$ by $\phi_{t}^{X}$ and $\varphi_{\tau_{i}}^{Y_{i}}$ with parameters $t$ and $\tau_{i}$, respectively. We set an initial point at the initial time $t=0$ as $q^{0} \in Q$. We also consider $m+1$ vector fields defined on the expanded configuration manifold $\bar{Q}=\mathbf{R} \times Q$ (3.18), and then their flows on $Q$ are obtained as

$$
\bar{\phi}_{t}^{\bar{X}}:=\left[\begin{array}{c}
t \\
\phi_{t}^{X}
\end{array}\right], \quad \bar{\varphi}_{\tau_{i}}^{\bar{Y}_{i}}:=\left[\begin{array}{c}
0 \\
\varphi_{\tau_{i}}^{Y_{i}}
\end{array}\right], \quad i=1, \ldots, m .
$$

Calculating the composite mapping of $(n+1)$ flows $(4.15)$ yields

$$
\bar{\Phi}:=\bar{\phi}_{t}^{\bar{X}} \circ \bar{\varphi}_{\tau_{1}}^{\bar{Y}_{1}} \circ \cdots \circ \bar{\varphi}_{\tau_{n}}^{\bar{Y}_{n}}=\left[\begin{array}{c}
t \\
\phi_{t}^{X} \circ \varphi_{\tau_{1}}^{Y_{1}} \circ \cdots \circ \varphi_{\tau_{n}}^{Y_{n}}
\end{array}\right]=\left[\begin{array}{c}
t \\
\Phi_{t}
\end{array}\right],
$$

where

$$
\Phi_{t}:=\phi_{t}^{X} \circ \varphi_{\tau_{1}}^{Y_{1}} \circ \cdots \circ \varphi_{\tau_{n}}^{Y_{n}}
$$

is the composite mapping of $n+1$ flows $\phi_{t}^{X}, \varphi_{\tau_{i}}^{Y_{i}}$. From (4.16), it turns out that the projection of $\bar{\Phi}$ onto $Q$ is equivalent to $\Phi_{t}$. Hence, by applying the idea of the integrating algorithm for scleronomous linear constraints defined on $Q[17,18]$ to $\mathbf{R} \times Q$ and considering projection of it onto $Q$, we can derive the following algorithm to calculate $n-m$ independent first integrals of completely integrable $A$-rheonomous affine constraints.

Algorithm 4.4. For the case where the A-rheonomous affine constraints (2.2) are $k(m<k<$ $n$ )th-order partially integrable, we can obtain $n-k$ independent first integrals of them by the following procedure.

Step 1. Set $m+1$ vector fields $X, Y_{1}, \ldots, Y_{m}$ of geometric representation for the $A$-rheonomous affine constraints (2.2). 
Step 2. By calculating iterated Lie brackets of $X, Y_{1}, \ldots, Y_{m}$, derive $k-m$ linearly independent vector fields $Y_{m+1}, \ldots, Y_{k}$ that satisfy

$$
C_{0}=\operatorname{span}\left\{Y_{1}, \ldots, Y_{m}, Y_{m+1}, \ldots, Y_{k}\right\}
$$

Step 3. For $X, Y_{1}, \ldots, Y_{k}$, derive linearly independent vector fields $Y_{k+1}, \ldots, Y_{n}$ that satisfy

$$
\operatorname{span}\left\{Y_{1}, \ldots, Y_{k}, Y_{k+1}, \ldots, Y_{n}\right\}=\mathbf{R}^{n}
$$

Step 4. Calculate flows of $X, \Upsilon_{1}, \ldots, \Upsilon_{n}: \phi_{t}^{X}, \varphi_{\tau_{i}}^{Y_{1}}, \ldots, \varphi_{\tau_{i}}^{\Upsilon_{n}}$.

Step 5. Combine $n+1$ flows derived in Step 4 as (4.17).

Step 6. Set $q=\Phi_{t}(\tau)$ and calculate the inverse function $\tau=\Phi_{t}^{-1}(q)$. Then, the last $n-k$ components of $\Phi_{t}^{-1}$ are first integrals of (2.2).

It is noteworthy that the differences between Algorithms 4.1 and 4.4 appear in Steps 2 and 6 in Algorithm 4.4, that is, we have to set $k-m$ vector fields that satisfies (4.18) and the number of independent first integrals obtained by Algorithm 4.4 is smaller than the ones obtained by Algorithm 4.1. Similar to Proposition 2.3, the existence of the inverse mapping $\Phi_{t}^{-1}$ at Step 5 in Algorithm 4.4 is guaranteed by the following proposition.

Proposition 4.5. Assume that the A-rheonomous affine constraints (2.2) are $k(m<k<n)$ th-order partially integrable. Then, there exists a time interval $I \subset \mathbf{R}$ and $\Phi_{t}(\tau)$ is a diffeomorphism at any time $t \in I$. That is, there exists its inverse mapping $\Phi_{t}^{-1}$.

Proof. This proposition can be proven by similar procedure to the proof of Proposition 2.7. The details are omitted.

Now, let us consider another simple example to check the availability of Algorithm 4.4 and Proposition 4.2 as follows.

Example 4.6. We now deal with the A-rheonomous affine constraints (3.41) on the 3dimensional configuration manifold (3.40), which are the same ones in Example 3.8. As seen in Example 3.8, we can find that (3.41) are 2nd-order partially integrable with $k=2$, there exists one independent first integrals, and hence we now calculate them by using Algorithm 4.4. One of the geometric representation for (3.41) is given by (3.42) and we set $Y_{1}:=Y$. Calculating iterated rheonomous brackets for (3.42), we have $C_{0}=\operatorname{span}\left\{Y_{1},\left\langle X, Y_{1}\right\rangle\right\}$, where

$$
\left\langle X, Y_{1}\right\rangle=\left[\begin{array}{c}
-q_{1} q_{3} \\
q_{2} q_{3} \\
0
\end{array}\right]
$$


Next, we set $Y_{2}:=\left\langle X, Y_{1}\right\rangle$ and calculate $Y_{3}$ satisfying (4.19) as

$$
Y_{3}=\left[\begin{array}{l}
1 \\
0 \\
0
\end{array}\right]
$$

and the flows of $X, Y_{1}, Y_{2}, Y_{3}$ can be obtained as

$$
\begin{array}{cc}
\phi_{t}^{X}=\left[\begin{array}{cc}
q_{1}^{0} e^{-(1 / 2) t^{2}+q_{3}^{0} t} \\
q_{2}^{0} e^{-q_{3}^{0} t} \\
q_{3}^{0}
\end{array}\right], & \varphi_{\tau_{1}}^{Y_{1}}=\left[\begin{array}{c}
q_{1}^{0} e^{q_{3}^{0}\left(e^{\tau_{1}}-1\right)-\tau_{1}} \\
q_{2}^{0} e^{-q_{3}^{0}\left(e^{\tau_{1}}-1\right)} \\
q_{3}^{0} e^{\tau_{1}}
\end{array}\right], \\
\varphi_{\tau_{2}}^{Y_{2}}=\left[\begin{array}{c}
q_{1}^{0} e^{-q_{3}^{0} \tau_{2}} \\
q_{2}^{0} e^{q_{3}^{0} \tau_{2}} \\
q_{3}^{0}
\end{array}\right], & \varphi_{\tau_{3}}^{Y_{3}}=\left[\begin{array}{c}
\tau_{3}+q_{1}^{0} \\
q_{2}^{0} \\
q_{3}^{0}
\end{array}\right],
\end{array}
$$

where $q^{0}=\left[\begin{array}{lll}q_{1}^{0} & q_{2}^{0} & q_{3}^{0}\end{array}\right]^{\top} \in Q$ is an initial point of the flows at the initial time. Combining (4.22) as (4.17), we have

$$
\Phi_{t}(\tau)=\phi_{t}^{X} \circ \varphi_{\tau_{1}}^{Y_{1}} \circ \varphi_{\tau_{2}}^{\gamma_{2}} \circ \varphi_{\tau_{3}}^{\gamma_{3}}=\left[\begin{array}{c}
\left(\tau_{3}+q_{1}^{0}\right) e^{-q_{3}^{0} \tau_{2}} e^{q_{3}^{0}\left(e^{\tau_{1}}-1\right)} e^{-(1 / 2) t^{2}+q_{3}^{0} e^{\tau_{1} t}} \\
q_{2}^{0} e^{q_{3}^{0} \tau_{2}} e^{-q_{3}^{0}\left(e^{\tau_{1}} t\right)} \\
q_{3}^{0} e^{\tau_{1}}
\end{array}\right],
$$

and from the equation $q=\Phi_{t}(\tau)$, we can calculate its inverse mapping:

$$
\Phi_{t}^{-1}(q)=\left[\begin{array}{c}
\log \frac{q_{3}^{0}}{q_{3}} \\
\frac{q_{3}(t+1)}{q_{3}^{0}}+\frac{1}{q_{3}^{0}} \log \frac{q_{2}}{q_{2}^{0}}-1 \\
\frac{q_{1} q_{2} q_{3} e^{(1 / 2) t^{2}}}{q_{2}^{0} q_{3}^{0}}-q_{1}^{0}
\end{array}\right] .
$$

Consequently, we can obtain one independent first integral of (3.41):

$$
h_{1}(t, q)=\frac{q_{1} q_{2} q_{3} e^{(1 / 2) t^{2}}}{q_{2}^{0} q_{3}^{0}}-q_{1}^{0}
$$

as the last component of (4.24).

\section{Physical Example: Pendulum with Time-Varying Elastic String}

In this section, we consider a physical example in order to verify the results obtained in the previous sections. We treat a pendulum with an elastic string, which is shown in Figure 12 and 


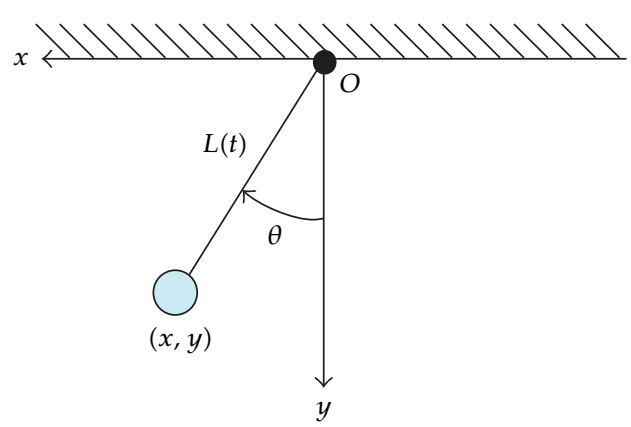

Figure 12: A single pendulum with a time-varying elastic string.

has already been dealt with in the first paper [38]. This system is composed of a weight and an elastic string. One end of the string is fixed to the ceiling and can swing around the fixed point. The weight is installed at the other end of the string. As depicted in Figure 12, we set the $x$-axis and the $y$-axis to the horizontal and vertical directions, respectively. We also set the origin of the coordinate system so that it corresponds to the fixed point of the string. Therefore, we consider the 2-dimensional configuration manifold as follows:

$$
Q=\left\{q=\left[\begin{array}{l}
x \\
y
\end{array}\right] \in \mathbf{R}^{2} \mid y \geq 0\right\}
$$

where $n=2$. The position of the weight in $Q$ is represented by $(x, y)$. In addition, the angle of the string in the clockwise direction measured from the $y$-axis is denoted by $\theta$. Now, we assume that the length of the string is changed as time goes by, that is, a time-varying elastic string, and we denote it by $L(t)$.

This system is undoubtedly subject to a constraint on the configuration variables as

$$
\sqrt{x^{2}+y^{2}}=L(t)
$$

We can easily see that since (5.2) does not contain any derivatives of the configuration variables, (5.2) is a rheonomous holonomic constraint. So, we can reduce the number of the configuration variables by replacing $(x, y)$ by $\theta$. However, we consider this system as a physical example which is subject to an integrable $A$-rheonomous affine constraint. Calculating time derivative of (5.2), we have

$$
x \dot{x}+y \dot{y}=\dot{L}(t) L(t) .
$$

Now, for the sake of simplicity, we set $N(t):=\dot{L}(t) L(t)$. Then, (5.3) can be represented as an $A$-rheonomous affine constraint defined on $Q$ :

$$
\underbrace{-N(t)}_{A(t)}+\underbrace{\left[\begin{array}{ll}
x & y
\end{array}\right]}_{B(q)}\left[\begin{array}{c}
\dot{x} \\
\dot{y}
\end{array}\right]=0,
$$


where $m=1$. Let us start with the above $A$-rheonomous affine constraint (5.4). One of geometric representation for the $A$-rheonomous affine constraints (5.4) can be derived as

$$
X=\left[\begin{array}{c}
0 \\
\frac{N(t)}{y}
\end{array}\right], \quad Y=\left[\begin{array}{c}
1 \\
-\frac{x}{y}
\end{array}\right]
$$

For the vector fields $X, Y$, we calculate a rheonomous bracket as

$$
\langle X, Y\rangle=\frac{\partial Y}{\partial t}+[X, Y]=\left[\begin{array}{c}
0 \\
\frac{N(t) x}{y^{3}}
\end{array}\right]-\left[\begin{array}{c}
0 \\
\frac{N(t) x}{y^{3}}
\end{array}\right]=\left[\begin{array}{l}
0 \\
0
\end{array}\right]
$$

and so we can see that all the iterated rheonomous brackets of $X, Y$ are 0 . Consequently, we obtain

$$
C_{0}=\operatorname{span}\{Y\}
$$

and hence

$$
\operatorname{dim} C_{0}=1, \quad \forall q \in Q, \forall t \in I:=\mathbf{R}
$$

holds. From the condition (c) in Theorem 3.1, it turns out that the A-rheonomous affine constraint (5.4) is completely integrable, and this fact coincides with the problem setting of this system. Furthermore, the condition (a) in Theorem 3.1, we can see that there exist one first integral of the $A$-rheonomous affine constraint (5.4). So, we next calculate it by using the integrating algorithm introduced in Section 4.1 (Algorithm 4.1). We set $Y_{1}:=Y$ and a vector field $Y_{2}$ that satisfies span $\left\{Y_{1}, Y_{2}\right\}=\mathbf{R}^{2}$ as

$$
Y_{2}=\left[\begin{array}{l}
1 \\
0
\end{array}\right]
$$

Next, the flows of $X, Y_{1}, Y_{2}$ can be calculated as

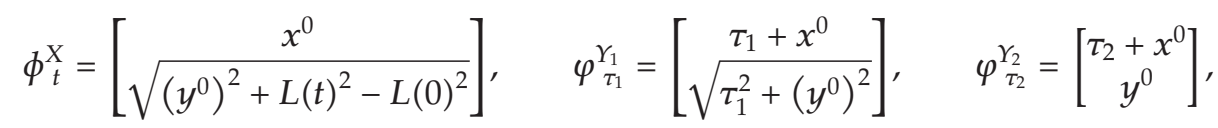

where $q^{0}=\left[\begin{array}{ll}x^{0} & y^{0}\end{array}\right]^{\top}$ is an initial point of the flows. Now, combining the flows (5.10) as (4.16), we then obtain

$$
\Phi_{t}=\phi_{t}^{X} \circ \varphi_{\tau_{1}}^{Y_{1}} \circ \varphi_{\tau_{2}}^{Y_{2}}=\left[\frac{\tau_{1}+x^{0}}{\sqrt{\tau_{1}^{2}+L(t)^{2}-L(0)^{2}+\left(\tau_{2}+y^{0}\right)^{2}}}\right] .
$$


From (5.11), we can calculate the inverse mapping of $\Phi_{t}$ as

$$
\Phi_{t}^{-1}(q)=\left[\begin{array}{c}
x-x^{0} \\
\sqrt{\left(x-x^{0}\right)^{2}+y^{2}-L(t)^{2}+L(0)^{2}}-y^{0}
\end{array}\right] .
$$

Therefore, the first integral can be obtained as the last component of (5.12):

$$
\sqrt{\left(x-x^{0}\right)^{2}+y^{2}-L(t)^{2}+L(0)^{2}}-y^{0}=0 .
$$

For the sake of simplicity, we set the initial values of $x, y, L(t)$ as $x^{0}=y^{0}=0, L(0)=0$. Thus, from (5.13), we can obtain

$$
x^{2}+y^{2}=L(t)^{2},
$$

and we can confirm that (5.14) corresponds to the rheonomous holonomic constraint (5.2).

\section{Conclusions}

In this paper, we have treated the $A$-rheonomous affine constraints from the aspects of foliation structures and integrating algorithms. We first investigate foliation structures of configuration manifolds with the A-rheonomous affine constraints for the three cases: complete integrability, partial integrability, and complete nonintegrability. Next, we have developed two kinds of integrating algorithms that can calculate independent first integrals of the $A$-rheonomous affine constraints in the complete integrability and partial integrability cases. Then, a pendulum with a time-varying elastic string as a physical example has been presented in order to show the effectiveness of the new results obtained in this paper.

It has turned out from the results that (a) for the completely and partially integrable $A$-rheonomous affine constraints, there exist not only time-invariant foliation structures but also time-varying ones in configuration manifolds, and this fact is similar to the ones for the scleronomous affine constraints [23], (b) for the completely nonintegrable $A$-rheonomous affine constraints, there do not exist any foliation structures, (c) independent first integrals of given $A$-rheonomous affine constraints can be calculated by using the integrating algorithms, (d) the rheonomous bracket plays important roles in analysis of foliation structures and derivation of integrating algorithms. These results obtained in this paper seem to be very useful for various research fields such as general mathematics and physics as well as control theory.

Throughout the first paper [38] and this second paper, we have considered a new class of constraints, the A-rheonomous affine constraints, which have been hardly researched so far, and we have developed some fundamental mathematical tools to analyze integrability/nonintegrability and geometric structures for the $A$-rheonomous affine constraints. Our future work on the $A$-rheonomous affine constraints are as follows: (i) theoretical analysis and control synthesis of nonholonomic kinematic/dynamic systems subject to $A$-rheonomous affine constraints, (ii) applications to the model reduction theory and constrained mechanical system theory, (iii) extensions to more general constraints, for example, fully rheonomous affine constraints of the form: $A(t, q)+B(t, q) \dot{q}=0$. In particular, 
(iii) seems to be quite difficult to solve because not only the rheonomous vector field $X$ but also the distribution $D$ contain the time variable $t$, that is, we have to deal with the timevarying distribution $D(t, q)$.

\section{Acknowledgment}

This work was partially supported by Kurata Grants.

\section{References}

[1] I. Ju. Neimark and N. A. Fafaev, Dynamics of Nonholonomic Systems, American Mathematical Society, 1972.

[2] Z. Li and J. F. Canny, Nonholonomic Motion Planning, Kluwer Academic Publishers, 1993.

[3] J. Cortés Monforte, Geometric, Control and Numerical Aspects of Nonholonomic Systems, vol. 1793 of Lecture Notes in Mathematics, Springer, Berlin, Germany, 2002.

[4] A. M. Bloch, Nonholonomic Mechanics and Control, vol. 24 of Interdisciplinary Applied Mathematics, Springer, New York, NY, USA, 2003.

[5] F. Bullo and A. D. Rewis, Geometric Control of Mechanical Systems, Springer Science, Business Media, 2004.

[6] C. Canudas de Wit and O. J. Sørdalen, "Exponential stabilization of mobile robots with nonholonomic constraints," Institute of Electrical and Electronics Engineers, vol. 37, no. 11, pp. 1791-1797, 1992.

[7] R. M. Murray and S. S. Sastry, "Nonholonomic motion planning: steering using sinusoids," Institute of Electrical and Electronics Engineers, vol. 38, no. 5, pp. 700-716, 1993.

[8] D. Tilbury, R. M. Murray, and S. S. Sastry, "Trajectory generation for the N-trailer problem using Goursat normal form," Institute of Electrical and Electronics Engineers, vol. 40, no. 5, pp. 802-819, 1995.

[9] C. Altafini, A. Speranzon, and B. Wahlberg, "A feedback control scheme for reversing a truck and trailer vehicle," IEEE Transactions on Robotics and Automation, vol. 17, no. 6, pp. 915-922, 2001.

[10] C. Fernandes, L. Gurvits, and Z. X. Li, "Near-optimal nonholonomic motion planning for a system of coupled rigid bodies," Institute of Electrical and Electronics Engineers, vol. 39, no. 3, pp. 450-463, 1994.

[11] X. S. Ge and L. Q. Chen, "Attitude control of a rigid spacecraft with two momentum wheel actuators using genetic algorithm," Acta Astronautica, vol. 55, no. 1, pp. 3-8, 2004.

[12] J.-M. Godhavn, A. Balluchi, L. S. Crawford, and S. S. Sastry, "Steering of a class of nonholonomic systems with drift terms," Automatica, vol. 35, no. 5, pp. 837-847, 1999.

[13] T. Mita, S. H. Hyon, and T. K. Nam, "Analytical time optimal control solution for a two-link planar acrobot with initial angular momentum," IEEE Transactions on Robotics and Automation, vol. 17, no. 3, pp. 361-366, 2001.

[14] M. D. Berkemeier and R. S. Fearing, "Sliding and hopping gaits for the underactuated acrobot," IEEE Transactions on Robotics and Automation, vol. 14, no. 4, pp. 629-634, 1998.

[15] M. D. Berkemeier and R. S. Fearing, "Tracking fast inverted trajectories of the underactuated Acrobot," IEEE Transactions on Robotics and Automation, vol. 15, no. 4, pp. 740-750, 1999.

[16] M. Reyhanoglu, A. van der Schaft, N. H. McClamroch, and I. Kolmanovsky, "Dynamics and control of a class of underactuated mechanical systems," Institute of Electrical and Electronics Engineers, vol. 44, no. 9 , pp. $1663-1671,1999$.

[17] A. Isidori, Nonlinear Control Systems, Communications and Control Engineering Series, Springer, Berlin, Germany, 3rd edition, 1995.

[18] S. S. Sastry, Nonlinear Systems, vol. 10, Springer, New York, NY, USA, 1999.

[19] H. Nijmeijer and A. van der Schaft, Nonlinear Dynamical Control Systems, Springer, New York, NY, USA, 1990.

[20] V. Jurdjevic, Geometric Control Theory, vol. 52 of Cambridge Studies in Advanced Mathematics, Cambridge University Press, Cambridge, UK, 1996.

[21] A. M. Bloch, M. Reyhanoglu, and N. H. McClamroch, "Control and stabilization of nonholonomic dynamic systems," Institute of Electrical and Electronics Engineers, vol. 37, no. 11, pp. 1746-1757, 1992.

[22] R. W. Brockett, "Asymptotic stability and feedback stabilization," in Differential Geometric Control Theory, vol. 27 of Progress in Mathematics, pp. 181-191, Birkhäuser, Boston, Mass, USA, 1983.

[23] T. Kai and H. Kimura, "Theoretical analysis of affine constraints on a configuration manifoldpart I: integrability and nonintegrability conditions for affine constraints and foliation structures of 
a configuration manifold," Transactions of the Society of Instrument and Control Engineers, vol. 42, no. 3, pp. 212-221, 2006.

[24] T. Kai and H. Kimura, "Theoretical analysis of affine constraints on a configuration manifold-part II: accessibility of kinematic asymmetric affine control systems with affine constraints," Transactions of the Society of Instrument and Control Engineers, vol. 42, no. 3, pp. 222-231, 2006.

[25] T. Kai, "Integrating algorithms for integrable affine constraints," IEICE Transactions on Fundamentals of Electronics, Communications and Computer Sciences, vol. E94-A, no. 1, pp. 464-467, 2011.

[26] T. Kai and K. Tamaki, "A near-optimal control approach to 3D ball-in-socket joint space robot models with initial angular momenta," Acta Astronautica, vol. 68, no. 11-12, pp. 1702-1711, 2011.

[27] T. Kai, H. Kimura, and S. Hara, "Nonlinear control analysis on kinematically asymmetrically affine control systems with nonholonomic affine constraints," in Proceedings of the 16th Triennial World Congress of International Federation of Automatic Control (IFAC '05), pp. 157-162, Czech Republic, July 2005.

[28] T. Kai, H. Kimura, and S. Hara, "Nonlinear control analysis on nonholonomic dynamic systems with affine constraints," in Proceedings of the 44th IEEE Conference on Decision and Control, and the European Control Conference (CDC-ECC '05), pp. 1459-1464, December 2005.

[29] T. Kai, "Affine constraints in nonlinear control theory," in Proceedings of the 3rd Workshop on Lagrangian and Hamiltonian Methods for Nonlinear Control, pp. 251-256, Nagoya, Japan, 2006.

[30] T. Kai, "Extended chained forms and their application to nonholonomic kinematic systems with affine constraints: control of a coin on a rotating table," in Proceedings 45th IEEE Conference on Decision and Control (CDC'06), pp. 6104-6109, usa, December 2006.

[31] T. Kai, "Derivation and analysis of nonholonomic hamiltonian systems with affine constraints," in Proceedings of the European Control Conference, pp. 4805-4810, Kos, Greece, 2007.

[32] T. Kai, "Generalized canonical transformations and passivity-based control for nonholonomic Hamiltonian systems with affine constraints: control of a coin on a rotating table," in Proceedings of the 46th IEEE Conference on Decision and Control(CDC '07), pp. 3369-3374, December 2007.

[33] W. Sarlet, F. Cantrijn, and D. J. Saunders, "A geometrical framework for the study of non-holonomic Lagrangian systems," Journal of Physics A, vol. 28, no. 11, Article ID 3253, 1995.

[34] D. J. Saunders, W. Sarlet, and F. Cantrijn, "A geometrical framework for the study of non-holonomic Lagrangian systems. II," Journal of Physics A, vol. 29, no. 14, pp. 4265-4274, 1996.

[35] M. de León, J. C. Marrero, and D. Martín de Diego, "Non-holonomic Lagrangian systems in jet manifolds," Journal of Physics A, vol. 30, no. 4, pp. 1167-1190, 1997.

[36] G. Giachetta, L. Mangiarotti, and G. Sardanashvily, "Nonholonomic constraints in time-dependent mechanics," Journal of Mathematical Physics, vol. 40, no. 3, pp. 1376-1390, 1999.

[37] F. Cantrijn, M. de León, J. C. Marrero, and D. Martín de Diego, "On almost-Poisson structures in nonholonomic mechanics. II. The time-dependent framework," Nonlinearity, vol. 13, no. 4, pp. 13791409, 2000.

[38] T. Kai, "Theoretical analysis for a class of rheonomous affine constraints on configuration manifoldspart I: fundamental properties and integrability/nonintegrability conditions," Mathematical Problems in Engineering, vol. 2012, Article ID 543098, 2012.

[39] S. Kobayashi and K. Nomizu, Foundations of Differential Geometry. Vol. I, Wiley Classics Library, John Wiley \& Sons, New York, NY, USA, 1996.

[40] S. Kobayashi and K. Nomizu, Foundations of Differential Geometry. Vol. II, Wiley Classics Library, John Wiley \& Sons, New York, NY, USA, 1996. 


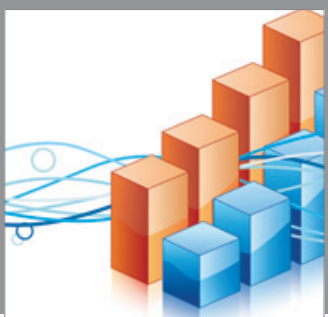

Advances in

Operations Research

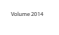

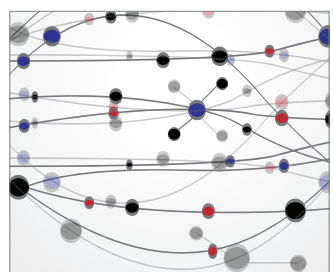

\section{The Scientific} World Journal
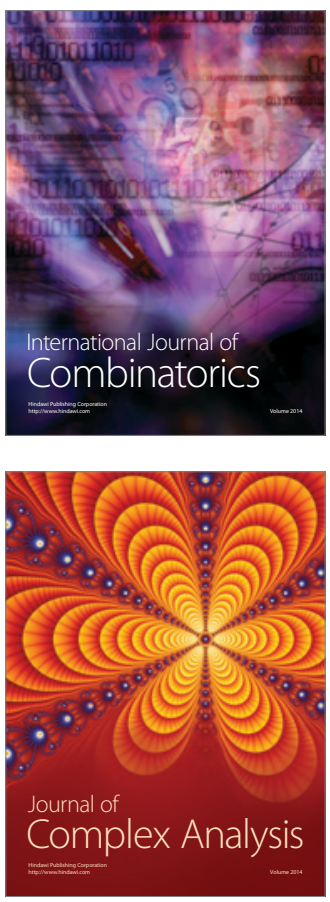

International Journal of

Mathematics and

Mathematical

Sciences
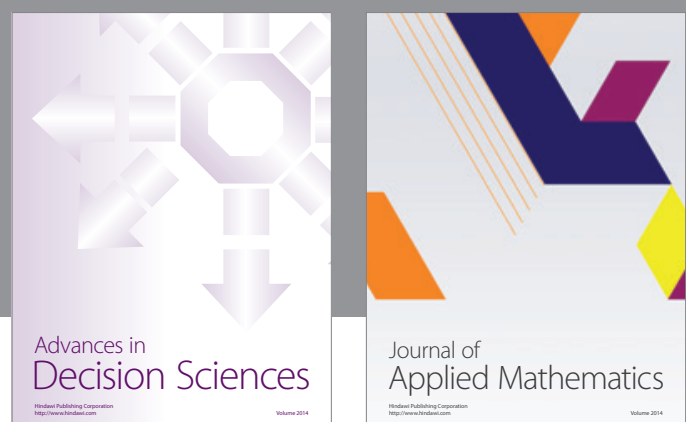

Journal of

Applied Mathematics
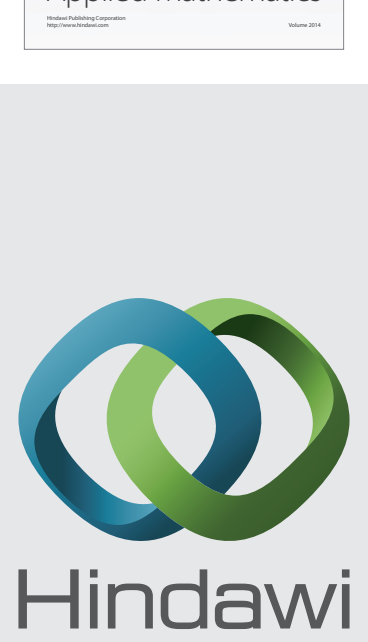

Submit your manuscripts at http://www.hindawi.com
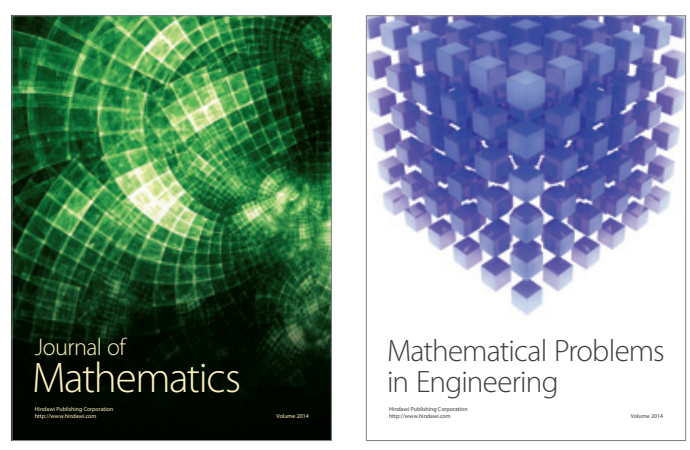

Mathematical Problems in Engineering
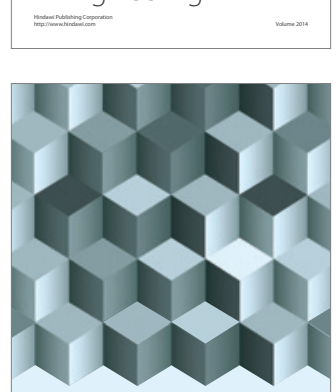

Journal of

Function Spaces
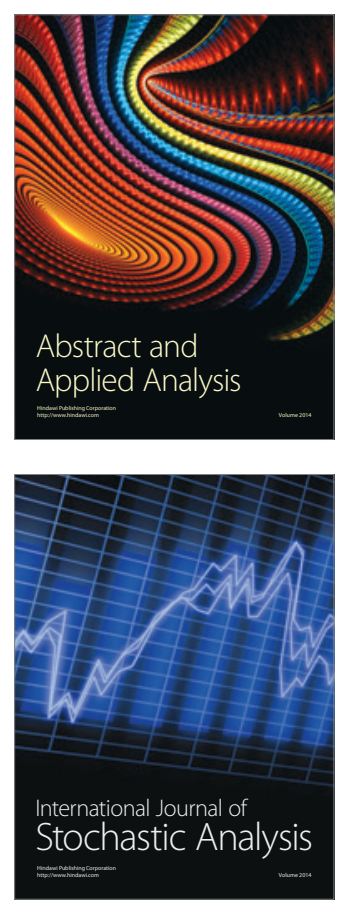

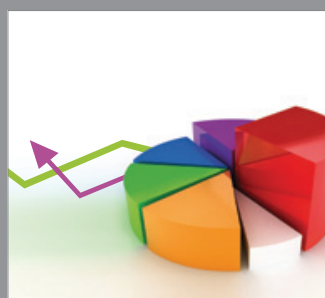

ournal of

Probability and Statistics

Promensencen
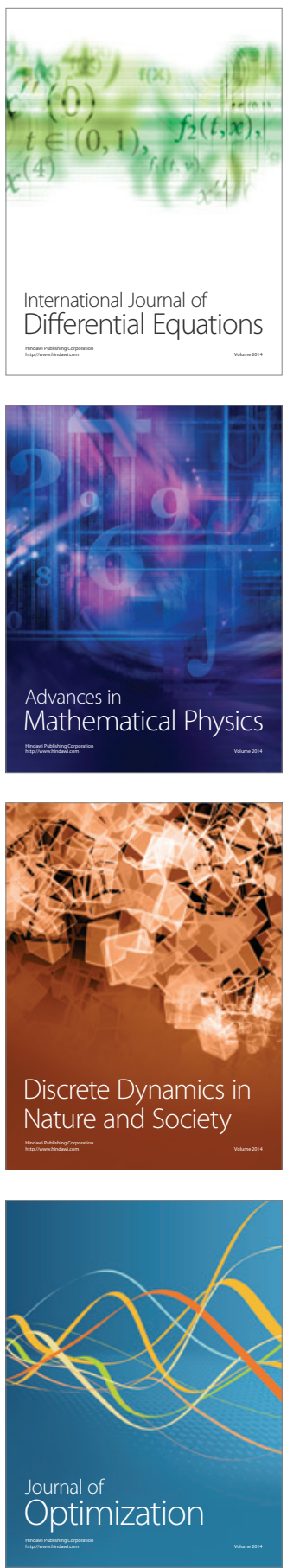